\title{
III.
}

\section{Der Begriff der Persönlichkeit bei Kant.}

\author{
Von
}

Daniel Greiner in Friedberg i. d. W.

I.

Die Persönlichkeit in transcendentalem Sinne.

Eine Erörterung des Begriffs der Persönlichkeit finden wir bei Kant zunächst in der ersten Auflage der Kritik der reinen Vernunft (Transc. Dialektik, 2. Buch, 1. Hauptstück) bei der Behandlung der Paralogismen der reinen Vernunft. Unter den Paralogismen der transcendentalen Psychologie, die hier der unerbittlichen Transcendentalkritik verfallen, befindet sich auch der der "Personalität", demzufolge das denkende Subjekt sich berechtigt glaubt, aus dem in verschiedenen Zeiten beharrenden Bewusstsein der numerischen Identität seiner Selbst auf die Existenz der SeeIe als Person zu schliessen. Kant zeigt die Unhaltbarkeit dieses Beweises in Hinblick auf dasjenige, was er (in derselben Auflage des Werkes) vorher (im 3. Abschnitte der Deduktion der reinen Verstandesbegriffe) über das Wesen der „reinen Apperception“ gelehrt hatte. Er verstand dort darunter „die durchgängige Identität seiner Selbst bei allen möglichen Vorstellungen", die dem empirischen Bewusstsein a priori zu Grunde liegt'). „Wir sind uns a priori der durchgängigen Identität unserer Selbst in Ansehung aller Vorstellungen ${ }^{2}$ ), die zu unserm Erkenntnis jemals

1) Rosenkranz und Schubert, J. Kant's Werke Band II, S. 105.

2) Ebenda 106 . 
gehören könnon, $:$ bewusst, als einer notwendigen Bedingung der Nöglichkeit aller Vorstellungen." Diese reine Apperception "gibt ein Principium der synthetischen Einheit des Nannigfaltigen in aller möglichen Anschauung an die Hand “ '). In der Sprache des seiner Kritik unterliegenden Paralogismus bedient sich Kant an der späteren Stelle hierfür des Ausdrucks Persönlichkeit: „Der Begriff der Persönlichkeit; sofern er blos transcendental ist, d. i. die Einheit des Subjekts; das uns übrigens unbekannt ist, in dessen Bestimmung aber eine durchgängige Verknüpfung durch Apperception ist" ").

Schärfere Bestimmungen über das Wesen der Apperception und somit auch über das Wesen der Persönlichkeit in dem hier festgelegten, d. h. in transcendentalem Sinne, gibt die Umarbeitung des betreffenden Abschnittes in der 2. Auflage ${ }^{3}$ ). Nach beiden Darlegungen stellt sich das Wesen dieser Apperception folgendermassen dar: die in der Zeit rasch aufeinanderfolgenden, wechselnden und sich drängenden Vorstellungen sind inhaltlich wio zeitlich gänzlich verschieden. Jede einzelne ist von dem Bewusstscin des Ich begleitet, sofern sie bewusst ist. Es wäre also ein einheitliches Bewusstsein nicht denkbar, wenn keine Verbindung, keine Einheit zwischen den einzelnen Bewusstseinsinhalten bestünde. „Ich würdo cin so vielfarbiges Selbst haben, als ich Vorstellungen habe, deren ich berusst bin ${ }^{* 6}$ ). Diese verbindende Einheit muss existieren, da ja thatsāchlich unser Bewusstsein einheitlich ist, und jedes vorstellende Subjekt sich in verschiedenen Zeiten und an verschiedenen Orten als identisch fühlt. Dieselbe kann aber nicht von aussen kommen, sie stammt nicht aus dem Nannigfaltigen der Erfahrung, denn dieses ist wechselnd und zerstreut, sie entspringt vielmehr der schöpferischen Einbildungskraft: Es ist dio allen bewussten Vorstellungen gemeinsame sie begleitende Vorstellung des "Ich denke ${ }^{\alpha}$. Das Ich ist der rote Faden, an dem sich allo dem Inhalt wie der Zeit nach so verschiedenen Vorstellungen aufreihen.
9) Ebenda.
9) Ebenda II, 237.
) Ebenda II, $730 \pi$.
7 Ebenda 730 l. 
Is fasst das Mannigfaltigo zusammen zu einor Einheit, die es uns möglich macht, unser Selbst als einheitlich $z \mathfrak{u}$ fühlen und $z \mathfrak{u}$ erkonuen. Es ist diese Einheit selbst. Alle Vorstellungen erhalten damit oino Beziehung auf ein einheitliches Subjekt, alles Mannigfaltige erhält durch die Bezogenheit auf das Ich Verbindung und Einheit. Diese Vorstellung des Ich gehört nicht der Sinnlichkeit an, sie ist „ein Actus der Spontaneität". Die reine Apperception oder auch die „ursprüngliche Apperception“, weil sie dasjenige Bewusstsein ist, was, „indem es die Vorstellung „ich denke“ hervorbringt, die alle anderen muss begleiten können, und in allem Bewusstsein ein und dasselbe ist, von keiner weiter begleitet werden

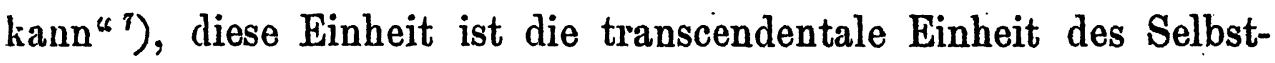
bewusstseins; sie allein macht Erkenntnis erst möglich, denn ohne sie wäre eine zusammenhängende Reihe von Vorstellungen, ein Denken unmöglich. Indem aber alle Vorstellungen, deren jede ihr eigenes Bewusstsein hat, einem allgemeinen Selbstbewusstsein unterliegen und untergeordnet werden, ist erst die Identität des Bewusstseins ermöglicht. „Ich bin mir des identischen Selbst bewusst, in Ansehung des Mannigfaltigen der mir in einer Anschauung gegebenen Vorstellungen, weil ich sie insgesammt meine Vorstellungen nenne, die eine ausmachen ${ }^{(8)}$ ).

2. In der Aufdeckung des „Paralogismus der Personalität“9 widerlegt nun Kant die Meinung der überlieferten metaphysischen Psychologie, dass man von der Thatsache einer Apperception oder der Persönlichkeit in transcendentalem Sinne auf die Existenz eines einheitlichen, d. h. persönlichen Denksubstrats (im Sinne einer "Seele") schliessen dürfe. Allerdings: „die Identität der Person [in dem bezeichneten Sinne] ist in meinem. Bewusstsein anzutreffen " ${ }^{10}$ ), aber es ist doch fraglich, ob diese empirische Beobachtung auch allgemein gültig ist, ob das Selbst wirklich identisch, diese verschiedenen Ich in verschiedenen Zeiten, Vorstellungen und Zuständen wirklich ein und dasselbe Ich sind? Ich selbst zwar

7) Ebenda II, 732.

8) Ebeuda II, 734.

9) Ebenda II, $290 \mathrm{ff}$.

10) Ebenda II, 291. 
erkenne in meinem Bewusstsein, in dem Ich das Beharrliche, aber „in dem Standpunkt eines Fremden können wir darum dieses noch nicht für gültig erklären, weil, da wir an' der Seele keine beharrliche Erscheinung antreffen, als nur die Vorstellung „Ich“, wolche sie alle begleitet und verknüpft, so können wir niemals ausmachen, ob dieses Ich (ein blosser Gedanke) nicht ebensowohl fliesse, als die übrigen Gedanken, die dadurch aneinandergekettet werden $\left.{ }^{(11}\right)$. Ein beharrliches numerisch identisches Ich als Subjekt des Denkens, eine Seele, lässt sich also gar nicht mit Sicherheit nachweisen. „Es ist also die Identität des Bewusstseins meiner Selbst in verschiedenen Zeiten nur eine formale Bedingung meiner Gedanken und ihres Zusammenhangs, berweist aber gar nicht die numerische Identität meines Subjekts, in welchem ungeachtet der logischen Identität des Ichs, doch ein solcher Wechsel vorgegangen sein kann, der es nicht erlaubt, die Identität desselben beizubehalten ${ }^{\alpha 3}$ ). Allerdings ist die Annahme eines solchen denkenden Subjekts notwendig als Grundlage und Voraussetzung alles Erkennens und Denkens, die ohne ein beharrliches Substrat nicht denkbar wären. Aber seine wirkliche Existenz lässt sich nicht beweisen. Um das $z \mathfrak{u}$ ermöglichen, müsste man das, was man beweisen möchte, vor allem voraussetzen, so käme man aus dem "Cirkel" nie heraus, da ich, "wenn ich das blosse Ich bei dem blossen Wechsel aller Vorstellungen beobachten will, kein anderes Correlatum meiner Vergleichungen habe, als wiederum mich selbst mit den allgemeinen Bedingungen meines Bewusstseins, so kann ich keine andere als tautologische Beantwortung geben, indem ich

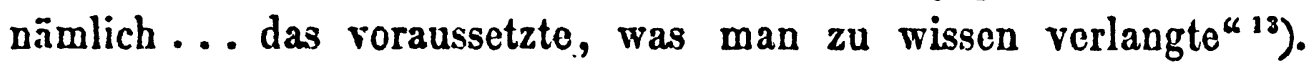
Was und dass dieses transcendentale Ich sei, lässt sich nicht sagen, da es die letzte Vorstellung ist, die ich in meinem Bowusstsein antreffe. Weil aber dieser Begriff zum praktischen Gebrauche durchaus unentbehrlich ist, so behält man ihn bei und gibt ihm seinen Namen. Kant nennt dieses transcendentale Unbekannte die transcendentale Persönlichkeit oder lieber mit dem all-

ii) Ebenda II, $29 \%$.

17) Elenda 11, 291.

17) Elsenda II, 293. 
gemcin gebriuchlichen Ausdruck: Secle. Eine genauere Erkenntnis dieses transcendentalen Substratums unsres Denkens erhalten wir damit nicht, es ist "sowenig Anschaunng als Begriff von irgend cinom Gegenstande, sondern die blosse Form des Bewusstseins, wolches beiderlei Vorstellungen begleiten und sie dadurch zu Erkeuntnissen erheben kann, soferne nämlich dazu noch irgend etwas Anderes in der Anschauung gegeben wird, welches zu einer Vorstellung von einem Gegenstande Stoff darreicht ${ }^{\text {(14}}$ ).

3. In der 2. Auflage der Kritik der reinen Vernunft hat Kant die Kritik der einzelnen Paralogismen zusammengezogen in die Widerlegung derjenigen Ansicht, die von dem Begriffe des Subjekts aus die Berechtigung darthun will, auf die Existenz der Seele als Substanz zu schliessen. Der Zusammenhang mit der den Begriff der Personalität betreffenden Erörterung in der ersten Auflage tritt dabei namentlich an zwei Stellen hervor II, 789: Die „Identität des Subjekts, deren ich mir in allen meinen Vorstellungen bewusst werden kann, betrifft nicht die Anschauung desselben, dadurch es als Objekt gegeben ist, kann also auch nicht die Identität der Person bedeuten“ u. s. w. und II,790 „ein Stein des Anstosses wider unsere ganze Kritik würde es sein, wenn es eine Möglichkeit gäbe, a priori zu beweisen, dass alle denkenden Wesen an sich einfache Substanzen sind, als solche also ... Persönlichkeit unzertrennlich bei sich führen". Als das Resultat der Untersuchung in beiden Auflagen lässt sich hinsichtlich des Begriffs der Personalität in dem bis jetzt erörterten Sinne etwa Folgendes hinstellen:

Diese transcendentale Persönlichkeit ist im Grunde nichts anderes als die empirische Persönlichkeit. Der Unterschied liegt nur in der Art und Weise der Betrachtung. Letztere vom Standpunkt der Transcendentalphilosophie aus betrachtet zeigt die Erstere. Das empirische Bewusstsein nimmt die Identität seiner Selbst als gegeben hin. Die kritische Philosophie untersucht auch hier die Grundlage und Gültigkeit der auf empirischem Wege gewonnenen Einsicht. Sie findet ein transcendentes Substrat des Denkens, ein

14) Ebenda 305 . 
Ding an sich, das weiter nicht bestimmbar ist. Sie führt damit an die Grenze der Sinnlichkeit, die sie damit einschränkt. Die Frage ist nur zurückgeschoben, nicht aber gelöst, einfach, weil sie nicht lösbar ist. Weiterhin ist damit auch die Grenze gegeben für den empirischen Verstandesgebrauch.

4. Grundlage beider, der empirischen wie der transcendentalen Persönlichkeit, ist das Bewusstsein. Woraus aber entspringt dies allgemeine Selbstbewusstsein? Kant führt es zurück auf die zusammenfassende, synthetische Arbeit des Verstandes, der die dem Vórstellungsvermögen sich aufdrängende Mannigfaltigkeit einheitlich verbindet durch die Vorstellung des Ich. Diese Verbindung „ist allein eine Verrichtung des Verstandes, der selbst nichts weiter ist, als das Vermögen a priori zu verbinden und das Mannigfaltige gegebener Vorstellungen unter Einheit der Apperception zu bringen, welcher Grundsatz der oberste in der ganzen menschlichen Erkenntnis ist" ${ }^{15}$ ). Und ferner: „So ist die synthetische Einheit der Apperception der höchste Punkt, an dem man allen Verstandesgebrauch, selbst die ganze Logik und nach ihr die Transcendentalphilosophie heften muss, ja dieses Vermögen ist der Verstand selbst ${ }^{* 16}$ ). Verfolgt man diesen Gedanken weiter, so ergibt sich für die Bestimmung des in Frage stehenden Begriffs: die Persönlichkeit, die Identitāt des Bewusstseins, d. i. „die Möglichkeit eines fortwährenden Bewusstseins in einem Subjekt, ist Produkt der synthetischen Arbeit des Verstandes. Der Besitz dieses „Vermōgens" also macht in letzter Linie das Wesen der empirischen Persōnlichkeit aus. Der Begriff der Persönlichkeit wäre demnach im Sinne Kant's zu bestimmen als ein mit Verstand begabtes Wesen. In diesem Sinne spricht sich auch die Einleitung zur Anthropologie aus: „dass der Mensch in seiner Vorstellung das Ich haben kann, erhebt ihn unendlich über alle andre auf Erden lebende Wesen. Dadurch ist er eine Person und vermöge der Einheit des Bewasstseins, bei allen Veränderungen des Bewusstseins, die ihm zastossen mögen, eine und dieselbe Person, d. i. ein ron Sachen, dergleichen die vernunftlosen Tiere sind, mit

19) Ebenda II, 734.

if Ebenda II, 733. 
denen man nach Belieben schalten und walten kann, durch Rang und Würde ganz unterschiedenes Wesen .... denn dieses Vermögen, nämlich zu denken, ist der Verstand “ ${ }^{17}$ ). Das auffassende Iuh (der Apprehension) hat der Mensch mit dem Tiere gemein, dagegen besitzt er allein das denkẹnde Ich (der Apperception), "das ihn von allen Tieren unterscheidet and sich selbst zum Gegenstande seiner Vorstellungen macht und sich der Verknüpfung seiner Vorstellungen bewusst ist. Das Letztere lässt sich nicht erklären. Es ist Spontaneität des Vorstellungsvermögens, woraus mit jenem verbunden Erkenntnisvermögen entspringt ${ }^{(18}$ ).

Somit ist der Mensch Persönlichkeit im empirischen Sinne vermöge seines Verstandes, der ihn fähig macht sich seiner selbst bewusst zu werden, die ihn umgebende Wirklichkeit und sein eigenes Selbst in einheitliche Begreiflichkeit zu bringen. Das dem Verstande entspringende Ichbewusstsein hebt ihn ab von der sonstigen Welt und hinaus über alle Sachen und die vernunftlosen Tiere. Er erhält damit eine Sonderstellung innerhalb des Naturganzen, der Sinnlichkeit, der es aber völlig unterstellt bleibt. Das Ichbewusstsein weist $\mathrm{ihm}$ einen gewissen Wert und eine $W$ ürde zu gegenüber allen Sachen, aber einen absoluten Wert erhält er damit noch nicht.

II.

Die moralische Persönlichkeit.

Als ein Resultat der bisherigen Untersuchung wird auch das herangetreten sein, dass Kant innerhalb der transcendentalen Erörterung den Begriff der Persönlichkeit nur nebenherverwendet, lediglich nur deshalb, weil unter den dort kritisierten metaphysischen Ansichten diejenige von der "Seele als Person" eine Hauptrolle spielte. Immerhin lässt er die Anwendung des Begriffes nach der bezeichneten Seite hin gelten und sucht nur dafür zu sorgen, dass er, jeder metaphysischen Bedeutsamkeit entkleidet und auf die Thatsache des verstandesmässigen Denkens unter der Mit-

17) Ebenda VII, 2. S. 11.

18) Reicke, Lose Blätter aus Kant's Nachlass II. Heft S. 91. 
wirkung der transcendentalen Apperception oder des Ichbewusstseins restringiert werde.

Ganz anders verhält es sich; wenn die Frage nach der moralischen Person des Menschen gestellt wird. Hier steht der Begriff der Persönlichkeit im Nittelpunkt der Untersuchung, die ganze Ethik Kants gravitiert um die endgiltige Bestimmung desselben, ja die Erhebung und Autorisierung des Menschen zur Persönlichkeit ist s. z. s. das letzte Wort der Kant'schen Philosophie. Mit der Behandlung dieses Punktes kommen wir daher erst recht zum Hauptthema unsrer Untersuchung. Bevor wir dieselbe in Angriff nehmen, sind zunächst aus den Ergebnissen der Kritik der reinen Vernunft noch einige Gedankengänge herauszuheben.

1. Durch die Vorstellung des Ich bringt der Verstand Einheit in das Manigfaltige des Bewusstseins, indem er es ordnet und unter Gesetze bringt. Nit seinen Begriffen und Kategorien überspinnt er gleichsam die ganze gegebene Welt der Gegenstïnde, wie mit einem Kleide, so dass er letztere niemals sehen kann, wio sie an sich sind, ohne die von ihm auferlegte Gesetzmässigkeit. Er dringt mit seinem Licht nur in die Welt der Erscheinungen; die Dinge an sich bleiben ihm unbekannt. Er kann eben „von seinen Begriffen niemals einen andern als empirischen Gebrauch machen", sie beziehen sich eben alle nur auf „Data zur möglichen Anschauung ${ }^{\alpha}$. Er bleibt in der Welt der Erfahrung. Wo er mit seinen Begriffen darüber hinaus zu kommen sucht, wagt er sich in gebrechlichem Fahrzeug auf ein uferloses Meer.

Obwohl der Verstand sich seine Begriffe selbstthätig schafft und er sich vermōge dieser Spontaneität zu einer Verstandeswelt erhebt, so bleibt er deshall doch innerhalb der Sinnenwelt haften. Ohne Anschaunngen sind die Begriffe völlig leer nsind ein blosses Spielwerk, es sei der Einbildungskraft oder des Verstandes" ${ }^{19}$ ). Diese aber kann ihm nur die Welt der Erscheinungen geben. So ist der Verstand abhängig von der Sinnenwelt. Seine eigenen Gesetze balten ihn gebunden innerhalb derselben. $\mathrm{Er}$ ist in gewisser Bezichung nur der Diener der Sinnlichkeit. Somit bleibt

i) Rosenkrauz II, 199. 
auch der Mensch, die empirische Persönlichkeit gebunden als Glied der Sinnlichkoit an das Naturganze, völlig unterworfen seinen ehernen Gesetzen. Auch als Intelligenz erhebt er sich nicht so über sie, dass er sich von den Banden der Sinnlichkeit irgend wie freimachen könnte.

2. Der Verstand bleibt also auf das Gebiet der möglichen Erfahrung beschränkt. Er hat es immer nur mit Erscheinungen zu thun, er kaun deshalb nie zu dem gelangen, was die Vernunft so unbedingt fordert, nämlich: systematische Einheit. Indem er zu dem Bedingten das Bedingende sucht, kommt er niemals zu einem Ende und Abschluss, denn jedes Bedingte fordert wieder seine Bedingung u.s. f. niemals erreicht er das Unbedingte und und somit die Totalität der Bedingungen. Um nun das auf allen Seiten offene Feld der Erfahrung zu begrenzen, schafft die Vernunft die Ideen, Begriffe, denen keine Anschauung gegeben werden kann. Sie gehen auf absolute Totalität in der Synthesis der Bedingungen und suchen "die synthetische Einheit, welche in der Kategorie gedacht wird, bis zum schlechthin Unbedingten hinauszuführen " ${ }^{20}$ ); "sie sind nicht willkürlich erdichtet, sondern durch die Natur der Vernunft selbst aufgegeben" ${ }^{21}$ ). Wenn sie auch weiter keine Erkenntnis bieten, so „können sie doch im Grunde und unbemerkt dem Verstande zum Kanon seines ausgebreiteten und einhelligen Gebrauches dienen, dadurch er zwar keinen Gegenstand mehr erkennt, als er nach seinen Begriffen erkennen würde, aber doch in dieser Erkenntnis besser und weiter geleitet wird “22). Oder mit andern Worten, sie haben keinen konstitutiven, sondern einen regulativen Gebrauch.

3. Die wichtigste dieser Ideen ist die der Freiheit. Die Kategorie der Causalität mit ihrem ehernen Gesetz von Ursache und Wirkung beherrscht das ganze Gebiet der Erfahrung. „Da aber“, deshalb „keine absolute Totalität der Bedingungen im Causalverhältnis herauszubekommen ist", wie es die Vernunft fordert, „so schafft sich die Vernunft die Idee von einer Spontaneität, die von

20) Ebenda II, 263.

21) Ebenda II, 263.

22) Ebenda II, 264. 
selbst anheben könne zu handeln, ohne dass eine andre Ursache vorangeschickt werden dürfe, sie wiederum nach dem Gesetz der Causalverknüpfung zur Handlung zu bestimmen ${ }^{\alpha 33}$ ). Dies ist die Idee der transcendentalen Freiheit. In praktischem Sinne bedeutet sie „Unabhängigkeit der Willkür von der Nötigung durch Antriebe der Sinnlichkeit“" ${ }^{24}$ ). Da nun die Gegenstände der Erfahrung nicht Dinge an sich sind, sondern Erscheinungen, so lässt sich nichts einwenden gegen die Annahme, dass es Wesen geben Könne, die als Dinge an sich, als , intelligible Wesen" das Vermögen haben, eine Reihe von Erscheinungen von selbst zu beginnen, mithin Freiheit, als „sensible“ Wesen, als Erscheinungen aber den Gesetzen der Causalität unterworfen sind. „Man würde von ihm ganz richtig sagen, dass es seine Wirkungen in der Sinnenwelt von selbst anfange, ohne dass die Handlung in ihm selbst anfängt", denn es untersteht als intelligibles Ding keiner Zeitbestimmung, „und dieses würde gültig sein, ohne dass die Wirkungen in der Sinnenwelt von selbst anfangen dürfen, weil sie in derselben . . . . nur als cine Fortsetzung der Reihe der Naturursachen möglich sind ${ }^{25}$ ). So enthält denn die Annahme von Freiheit und Naturnotwendigkeit in demselben Wesen rom Standpunkte der Transcendentalphilosophie aus keinen Widerspruch. Die Möglichkeit oder gar die Wirklichkeit dieser Idee ist jedoch damit noch nicht erwiesen.

4. Für den spekulativen Gebrauch unserer Vernunft bedeuten die Ideen keine Erweiterung unsrer Erkenntnisse. Desto grössere Wichtigkeit zeigen sie dagegen für den praktischen Gebrauch. „Praktisch ist alles, was durch Freiheit möglich ist ${ }^{4}$. Die praktische Freiheit nun kann durch Erfahrung bewiesen werden. „Denn nicht bloss das, was reizt, d.i. die Sinne unmittelbar afficiert, bestimmt die menschliche Willkür, sondern wir haben ein Vermögen, durch Vorstellungen von dem, was selbst auf entfernte Art nützlich oder schädlich ist, die Eindrücke auf unser sinnliches Begehrungsvermögen zu überwinden, diese Ueberlegungen aber . . . . beruhen

23) Ebeada II, 413.

20) Ebeada.

23) Ebenda II, 425.

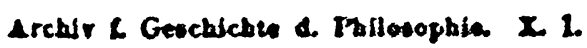


auf der Vernunft" ${ }^{26}$ ). „Diese giebt daher auch Gesetze, welche Imperative, d. i. objoktive Gesetze der Freiheit sind, und welche sagen, was geschehen soll, ob es gleich, vielleicht nie geschieht, und sich darin von Naturgesetzen, die nur von dem handeln, was geschieht, unterscheiden, weshalb sie auch praktische Gesetze genannt werden ${ }^{37}$ ). So erhebt sich neben dem Reich des Seins ein anderes Reich des Sollens, neben dem „Manigfaltigen des Bewusstseins“, ein „Manigfaltiges der Begehrungen“. Neben „der Causalität, nach der man sich denkt, das etwas geschieht", giebt es eine andere, „wo man von etwas sagen kann, es hätte geschehen sollen, ob es zwar in allen Bestimmungsgründen, die Erfahrung geben kann, unwandelbar ausgemacht ist, dass es nicht geschehen ist, noch sein kann, und das ist das moralischpraktische Sollen; was daher absolut ist, und das Können schlechthin vorausgesetzt $\left.{ }^{(28}\right)$.

Nicht nur ein rezeptives leidendes Vermögen findet sich im Menschen, sondern auch ein Vermögen der Freiheit. Dies Vermögen beruht in der Vernunft. „Diese als reine Selbstthätigkeit ist sogar noch darin über den Verstand erhoben, dass, obgleich dieser auch Selbstthätigkeit ist, und nicht wie der Sinn blosse Vorstellungen enthält, die nur entspringen, wenn man von Dingen affiziert (mithin leidend) ist, er dennoch aus seiner Thätigkeit, keine anderen Begriffe hervorbringen kann, als die, welche blos dazu dienen um die sinnlichen Vorstellungen unter Regeln zu bringen und sie dadurch in einem Bewusstsein zu einigen, ohne welchen Gebrauch der Sinnlichkeit er gar nicht denken würde. Dahingegen die Vernunft unter dem Namen der Ideen eine so reine Spontaneität zeigt, dass er dadurch über alles, was ihm Sinnlichkeit nur liefern kann, hinausgeht, und ihr vornehmstes Geschäft darin beweist, Sinnenwelt und Verstandeswelt von einander zu scheiden " $\left.{ }^{29}\right)$. Indem sie das Wollen frei macht von der Bedingung der empirischen $W^{\top}$ elt, errichtet sie eine Ordnung der Dinge,

26) Ebenda II, $618 \mathrm{f}$.

27) Ebenda II, 618.

28) Reicke, a. a. O. II, 75.

29) Rosenkranz, a. a. 0 . VIII, $85 \mathrm{f}$. 
die die Sinnlichkeit und Welt der Causalität weit überragt, eine intelligible, übersinnliche Welt, in der das Gesetz der Freiheit, das Sittengesetz, Naturgesetz ist.

Glied dieser übersinnlichen Welt ist die moralische Persönlichkeit. Diesen Begriff zu erläutern und zu bestimmen ist Aufgabo des Folgenden:

A.

„Der Mensch ist ein bedürftiges Wesen sofern er zur Sinnenwelt gehört, und soferne hat seine Vernunft allerdings einen nicht abzulehnenden Auftrag von Seiten der Sinnlichkeit, sich um das Interesse derselben zu bekümmern und sie praktische Maximen, auch in Absicht auf die Glückseligkeit des Lebens zu machen. Aber er ist doch nicht so ganz Tier, um gegen alles, was Vernunft sagt, gleichgültig zu sein, und diese blos zum Werkzeuge der Befriedigung seines Bedürfnisses als Sinnenwesen zu gebrauchen, denn im Werte über die blosse Tierheit, hebt ihn das gar nicht, dass er Vernunft hat, wenn sie ihm nur zum Behuf desjenigen dienen soll, was bei Tieren der Instinkt verrichtet, . . . er bedarf also freilich ... der Vernunft, um sein Wohl und Wehe jederzeit in Betracht zu ziehen, aber er hat sie überdies noch zu einem höheren Behuf, nämlich das, was an sich gut oder böse ist, und worüber reine, sinnlich gar nicht interessierte Vernunft nur allein urteilen kann, nicht allein mit in Ueberlegung zu nehmen, sondern dieso Beurteilung gänzlich von jener zu unterscheiden und sie zur obersten Bedingung des letzteren zu machen ${ }^{\alpha 30}$ ). Diese Worte der Kritik der praktischen Vernunft sind sehr bezeichnend für das ganze Denken unsres Philosophen. Sie zeigen klar, welches Ziel sich als letztes dem Philosophen ergibt, sie weisen dio ganze Spitze seines Systems: den Menschen über die sinnliche Bedingtheit, die "Tierheit in ihm hinauszuheben und ihn zum Glied einer höheren geistigen Wirklichkeit zu machen. Die Thatsache einer unsinnlichen Wirklichkeit wird konstatiert, ein Reich der Sitten, des Guten und Bösen, mit eigenen, von aller sinnlichen Bedingt-

29. 2. 2. 0. Vill, $181 \%$. 
heit freien Gesetzen, ein Reich des Sollens an der Grenze des Reichs des Seins.

1. Man hat noch zunächst von Pflicht, die in dem Begriff des Sollens liegt, abzusehen. Sollen ist hier Wollen. Ueber dem rein vitalen Begehrungsvermögen, neben dem auf Selbstwohl und Glückseligkeit gerichteten, dunklen Drang steht das vernünftige, bewussto Wollen das „obere Begehrungsvermögen", das durchaus nicht mit dem "unteren" zusammengeworfen werden darf. Dieses Wollen bezieht sich auf Gegenstände nur sofern es dieselben hervorbringen will, und zwar nach Gesetzen; es ist das hervorbringende Denken. Insofern diesem Wollen im Menschen Triebe und Neigungen entgegenstehen und es in seiner Wirksamkeit behindern, wird es zum Sollen. . Somit ist das. Sollen eine Nötigung des Wollens, d. i. „das Verhältnis der objektiven Gesetze zu einem nicht durchaus guten Willen wird vorgestellt als die Bestimmung des Willens eines vernünftigen Wesens zwar durch Gründe der Vernunft, denen aber dieser Wille seiner Natur nach nicht notwendig folgsam ist" ${ }^{31}$ ).

2. Allgemeinheit und Notwendigkeit muss dieses Sollen enthalten in seinen Gesetzen. Um allgemein gültig zu sein, dürfen seine Grundsätze und Bedingungen nicht der Erfahrung entnommen werden; denn diese ist zu sehr dem Wechsel und der subjektiven Willkür preisgègeben. Ein so allgemeiner Massstab von a priorischer Gültigkeit kann nur der Vernunft entnommen werden. Nur dann ist die Gefahr ausgeschlossen, dass ein Sittengesetz auf empirischer Grundlage errichtet wird, was den Tod desselben bedeuten würde, weil damit seine Allgemeinheit in Frage gestellt wäre. Es darf also nicht von der Erfahrung abgelesen werden. Rücksicht auf Triebe, Neigungen, Begehren, Lustgefühle, wenn auch der feinsten Art, Glückseligkeit muss unbedingt ausser Berechnung gelassen werden. Diese Forderung stellt mit aller Schärfe der Lehrsatz I der Kritik der praktischen. Vernunft (I. Teil, 1. Buch, 1. Hauptstück): „Alle praktischen Principien, die ein Objekt (Materie) des Begehrungsvermögens, als Bestimmungsgrund des Willens,

31) Ebenda VIII, 36. 
voraussetzen, sind insgesamt empirisch und können keine prakti-

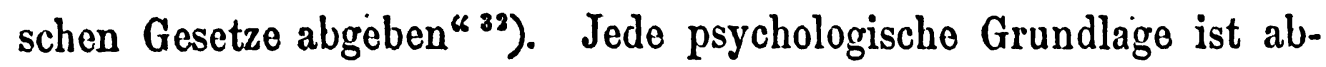
gewiesen. „Was aus der besonderen Naturanlage der Menschheit ... ja sogar, wo möglich aus einer besonderen Richtung, die der menschlichen Vernunft eigen wäre... abgeleitet wird, das kann zwar eine Maxime aber kein Gesetz abgeben, ein subjektives Prinzip nach dem wir handeln zu dürfen Neigung und Hang haben, aber nicht ein objektives, nach welchem wir zu handeln angewiesen wären, wenngleich aller unser Hang, Neigung, Naturrichtung dawider wäre $\left.{ }^{43}\right)$. Damit ist auch jede anthropologische und theologische Begründung ausgeschlossen. Der Forderung der Allgemeinheit kann nur dann Genüge geleistet werden, wenn die Grundsätze nur der Vernunft entnommen werden mit Ausschluss jeder auch

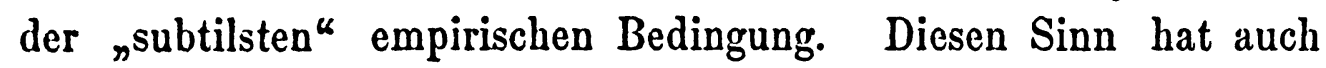
die oft wiederkehrende und viel augegriffene These, das Sittengesetz müsse „für vernünftige Wesen überhaupt gelten" ${ }^{34}$ ).

32) Ebenda VIII, 128.

33) Ebenda VIII, 53 f. 35. 128. 144.

34) So namentlich Cohen in „Kants Begründung der Etbik“, Berlin 1877 Seite $137 \mathrm{ff}$. Grund dieser, in der That aufallenden und zum Irreführen gocigneten Verallgemeinerung "ist "von dem blos Natürlichen, von dem physisch Anthropologischen, sollte der Begriff einer äthischen Realität nicht abgeleitet werden ... Dieser Grundbegriff wurde um so schärfer hervorgehoben, wenn der Blick nicht blos von allem, was in der Menschenwelt passiert, abgelenkt, sondern positir zu einer anderen, sinnlich unbestimmten Art von Realität erboben wurde" (137). Diesen Gedanken spricht auch Kant aus. Das Sittengesetz erbālt damit sofort eine höbere Realitāt, so dass „selbst weṇn Menschen nicht wãren, doch das Sittliche sein müsste". Cohen spottet dabei über Schopenbauer, der das „aprioristische Vorurteil zum Unglaublichen, ja, bei allem Respekte, zum Lācherlichen gesteigert habe*, wenn er sagt: „Man kann sich des Verdachtes nicht erwehren, dass Kant dabei ein wenig an die lieben Englein gedacht, oder doch auf deren Beistand in der Ueberzeugung des Lesers gerechnet habe*. (S. S. [Reclam] III, 512.) Indes ist dieser Verdacht gar nicht so unbegrūndet, wie folgende Stellen zeigen: "Es schränkt sich also nicht blos auf Jenschen ein, sondern geht auf alle endlichen Wesen, die Vernunft und Wille haben" u. s.f. (VIII, 38) und: "Die sittliche Stufe, worauf der Jensch (aller unsrer Einsicht nach auch jedes vernünftige Geschopf) stebt u. s. f." (VIII, 132.) Solche Stellen lassen schon den Verdacht auflommen, dass Kant hierbei an die Geltung des Sittengesetzes auch für vernünftige Wesen ausser dem Yenschen gedacbt habe. Doch bat dies immer nur bjpothetiscte Bedeutung. Kant entfernt sich damit nicht rom 
Die Erfahrung „die Muttor alles Scheins“ ist damit aus der Begründung des Sittengesetzes ausgeschlossen; sie kann nur „das Gomüt zwischen Bewegursachen, die sich unter kein Prinzip bringon lassen, die nur sehr zufällig zum Guten, öfters aber auch zum

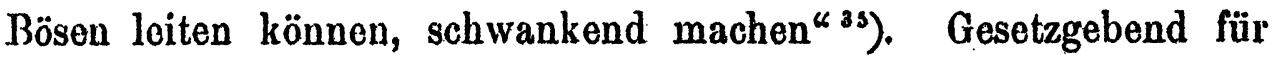
den Willen kann also nur Vernunft sein. Sie ist „causa originaria des Willens. Diese causalitas originaria der reinen Vernunft besteht eben in ihrer Allgemeinheit und Unabhängigkeit von empirischen Gründen ${ }^{36}$ ).

3. Wille aber ist „das Vermögen nach der Vorstellung der Gesetze, d. i. nach Prinzipien zu handeln ${ }^{37}$ ), er muss von jedem Gegeustand der Handlung abstrahieren, er geht blos auf die Handlung, nicht auf das Objekt derselben und handelt nur nach Regeln. Dadurch unterscheidet er sich vom Begehren: „Wollen ist etwas mit Bewusstsein durch seine eigene Handlung begehren " ${ }^{38}$ ). Willen können demzufolge nur vernünftige Wesen haben. Es ist „unbedingte Gesetzgebung der reinen praktischen Vernunft", ja die praktische Vernunft selbst ${ }^{39}$ ). Soll dieser Wille nun allgemein nötigend sein, soll er sich „als praktisches Gesetz zur allgemeinen Gesetz-

Standpunkt der Kritik der r. Vft., wie Schopenhauer meint (cfr. Hegler, Die Psychologie in Kant's Ethik (Freiburg 91) S. 140). Hauptzweck bei Einführung des Begriffs des „vernünftigen Wesens“ ist, dem Sittengesetz eine höhere Realität zu sichern; damit aber wird auch der Mensch „in eine höhere Sphäre, in die Gemeinschaft mit anderen vernünftigen Wesen emporgehoben" (Hegler a. a. 0. 141). Auch Cohen hebt diesen andern Gesichtspunkt hervor: „Andrerseits aber soll die Aufstellung der besonderen Art einer ethischen Realität gerade ... zu einer Erhöhung des Begriffs derselben (der Menschheit führen . . . es ist damit eben der Begriff des Menschen zu dem des vernünftigen Wesens erhöht; die Tierheit in ihm ist eliminiert: Die Freiheit ist zu seinem Gattungscharakter geworden." Cohen a. a. 0. 138).

35) Rosenkranz VIII, 34.

36) Reicke, a. a. 0. II, 27.

$\left.{ }^{37}\right)$ Rosenkranz VIII, 36 cf. 55; 80.

35) Reicke II, 9.

${ }^{39}$ ) „Nur ein vernünftiges Wesen hat das Vermögen nach Gesetzen, d. i. nach Prinzipien zu handeln oder einen Willen. Da zur Ableitung von Handlungen Vernunft erfordert wird, so ist der Wille nichts anderes als praktische Vernunft." (VIII, 36.) Doch erscheint in andern Stelle der Wille als etwas von der Vernunft Verschiedenes, von ihr Abhängiges, das auch andern Bestimmungsgründen zugänglich ist. cf. Hegler a. a. $0.158 \mathrm{ff}$. 
gebung qualifizieren ${ }^{40}$ ), so kann er nur als reiner Wille diese Allgemeinheit beanspruchen.

Wie von der Anschauung nur die reine Anschaunng als a priori, als allgemein, objektiv, notwendig sich erwies, und sich so die reine Form der Anschauung ergab, so kann auch nur die Form des Wollens, die Form seiner Gesetzmässigkeit, sich zur „allgemeinen Gesetzgebung schicken“. Diese „allgemeine Gesetzmässigkeit überhaupt", welchè dem Willen zum Prinzip dienen kaun und soll, erhalte ich, wenn ich von aller Beziehung des Wollens auf irgend einen Gegenstand absehe. „Nun bleibt von einem Gesetz, wenn man alle Materie, d. i. jeden Gegenstand als Bestimmungsgrund, davon absondert, nichts übrig als die blosse Form einer Gesetzgebung ${ }^{1}$ ). Nur diese aber kann objektives Gesetz für alle vernünftigen Wesen $\operatorname{sein}^{{ }^{2}}$ ). Denn, wenn ein vernünftiges Wesen sich seine Maximen als allgemeine, praktische Gesetze denken soll, so kann es sich dieselben nur als solche Prinzipien denken, die nicht der Materie, sondern blos der Form nach, den Bestimmungsgrund des Willens enthalten". (Lehrsatz III. Krit. d. pr. Vft. I. Teil, 1. Buch, 1. Hauptstück.)

Diese blosse gesetzgebende Form des Willens ist das Grundgesetz der reinen praktischen Vernunft. Mit Beziehung auf den Jenschen, der auch andern Bestimmungsgründen, als denen der Vernunft allein folgt, bedeutet es ein Sollen, seine Form ist der kategorische Imperativ, der „das' Verhältnis objektiver praktischer Gesetze des Wollens überhaupt zu der subjektiven Unvollkommenheit des Willens dieses oder jenen vernünftigen Wesens, z. B. des menschlichen Willens ausdrückt ${ }^{42}$ ). Seine Formel, das Grundgesetz der reinen praktischen Vernunft, lautet nach der Formulierung in der Kritik der praktischen Vernunft: „Ilandle so, dass dio Marime deines Wollens jederzeit zugleich als Prinzip einer allgomeinen Gesetzgebung gelten könne" ").

97) Rosenkranz VIII, 137.

ii) Ebenda VIII, 136 cf. 139.

-7) Ebenda VIII, 125. 139. 156. 196. 30f. u. a. m.

4) Ebenda VIII, 36.

1) Ebeada VIII, 141. 
4. Dieses Prinzip des Sittengesetzes kann alloin der Vernunft entspringon, da os jeder empirischen Grundlage entbehrt, mithin oin Grundsatz a priori ist, nder auf keine, weder auf reine noch cmpirische Anschauung gegründet ist ${ }^{45}$ ). Er kann nur auf reine praktische Vernunft zurückgehen, da er ja keine Bestimmungen übor das enthält, was geschieht, sondern dessen, was geschehen soll. So ergibt sich dann als Schlussfolgerung: „Reine Vernunft ist für sich allein praktisch und gibt dem Menschen ein allgemeines Gesetz, welches wir das Sittengesetz nennen ${ }^{646}$ ). Das ist keine Hypothese, sondern wirklich, „wie das Bewusstsein des moralischen Gesetzes es ausweist ${ }^{(47}$ ), und das Bewusstsein dieses Gesetzes ist ein "Faktum", das „unleugbar"48) ist. Aber woher und wie das komme, zu fragen, ist gänzlich nutzlos. Es ist da. „Auch ist das moralische Gesetz gegeben gleichsam als ein Faktum der reinen praktischen Vernunft, dessen wir uns a priori bewusst sind... gesetzt auch, dass man auch in der Erfahrung kein Beispiel, da es genau befolgt wäre, auftreiben könnte. Also kann das moralische Gesetz durch keine Deduktion ... bewiesen und also, wenn man auch auf die apodiktische Gewissheit Verzicht thun wollte, durch Erfahrung bestätigt und so a posteriori bewiesen werden und steht dennoch für sich fest" ${ }^{49}$ ). „Es ist res facti, dass dieses Gesetz in uns und zwar das oberste in uns ist" ${ }^{60}$ ).

45) Ebenda VIII, 142.

46) Ebenda.

47) Ebenda VIII, 260.

48) Ebenda VIII, 140. 142. 143. 157. 163. 173 u. a. m.

49) Ebenda VIII, 163.

50) Reicke, a. a. 0. II, 8. Diese Sätze, die alle besagen: Das Sittengesetz ein Faktum, sind leicht irreführend. Dieser Ausdruck scheint bedenklich an das Metaphysische zu streifen, das Kant in der Kritik der reinen Vernunft so energisch ein für allemal abgewiesen hat. Doch darf, da weder an ein metaphysisches Angebinde der gütigen Gottheit, noch an ein Angeborensein der sittlichen Grundsätze, an etwas Substantielles gedacht werden, wie Schopenhauer argwölınt. „Was soll man sich bei diesem seltsamen Ausdrucke deuken? Das Faktische wird sonst überall dem aus reiner Vernunft Erkennbaren entgegengesetzt." Scheint da Kant's "Fundament der Ethik nicht substantieller werden zu wollen"? (S. W. Reclam Bd. III, 525.) Es scheint nur so. Kant hat später den Ausdruck abgeschwächt in "gleichsam ein Faktum", das nachher immer wieder begegnet. Es ist gleichbedeutend mit der in der Grund- 
Ein wesentliches Merkmal des Sittengesetzes ist somit: sein Ursprung aus dor reinen praktischen Vernunft. Das besagt aber Unabhängigkeit von allen empirischen Grundlagen und Motiven, somit Freiheit von allem Naturmechanismus, d. i. Freiheit im negativen Sinne.

5. Indem die Vernunft dem Willen Gesetze vorzuschreiben vermag, indem sie ihm im Sittengesetz eine formale Regel seines Verhaltens gibt, die von keiner Erfahrung abgelesen ist, erweist er sich damit als selbstgesetzgebend, als autonom, denn der Wille

legung und der Kritik der praktischen Vernunft oft sich findenden Berufung auf den gemeinen Jenschenverstand z. B. , das rorher genannte Faktum ist unleugbar. Nan darf nur das Orteil zergliedern, welches die Menschen über die Gesetzmāssigkeit ihrer Handlungen fallen “ (VIII, 143). Allerdings klingen manche Stellen sehr verdächtig, wie z. B. die in einer Anmerkung in der Rel. inverhalb der Grenzen der reinen Vernunft: „Wäre dieses Gesetz nicht uns gegeben, wir würden es als ein solches durch keine Vernunft herausklügeln, oder der Willkür anschwatzen. Und doch ist dieses Gesetz das einzige, das uns der Unabhängigkeit unserer Willkür von der Bestimmung durch alle andern Triebfedern unsrer Freiheit ... bewusst " macht" (XI, 28). Was lāsst sich gegen so deutliche Stellen noch einwenden?

Zunächst ist zu bemerken, dass unter Sittengesetz nur das formale Sittengesetz gemeint ist. Nicht etwa einzelne sittliche Grundsātze sind in uns als Faktum, sondern allein die Form des Sittengesetzes lebt in unserm Bewusstsein. Daraus ergibt sich eine Lösung der Schwierigkeît, die Kant selbst andeutet, indem er das Faltum des Sittengesetzes vergleicht mit der Wirklichbeit der formalen Grundsātze der theoretischen Vernunft. "Wir bönnen uns reiner praktischer Gesetze bewusst werden, ebenso wie wir uns reiner theoretischer Grundsātze bewusst sind" (VIII, 140). Wie die reinen Formen des Raums und der Zeit aller Anschauung a priori vorhergehen, so geht das formale Sittengesetz allem IIandeln voraus; dies zeigt sich, ,sobald wir Maximen entrerfen". "Die Form gibt eine gesetzmãssige Verfabrungsweise... man kann wohl nicht sagen: Raum, Zeit, Kategorien, kategorischer Imperativ sind ursprüngliche Elemente des menschlichen Geistes ... etwas vor aller Thätigkeit feststehendes Substantielles, während sie genauer nur das Gesetzmässige in einer Thätigkeit bezeichnen" (Hegler a. a. 0. 92). "Das Gesetz ist das einzige Faltum der teinen Fernunft, die sich dadurch als ursprünglich gesetzgelend (sic rolo sic jubeo) ankündigt" (VIII, 143). Somit hat es keine Schwierigkeit mehr, wenn Kant das Sittengesetz ein Faktum nennt, es ist keine ,rerbürgte Thatsache des Bewusstseins" (Cohen), sondern es hat objektive Realitat als Idee mit Berug auf mügliche Erfahrung. Als Ideo ist die Idee des Sittengesetzes ebenso unvermeidliche Thatsache des Bewusstseins resp. der Vernunft als die anderen Ideen auch. Es erbebt sich an der Grenze der Erfahrung "als Ausgangspunkt für die ethische Zergliederung“. 
ist die praktische Vernunft. Er ist somit Gesetzen unterworfen, die or solbst aufstellt, die er aus seinem eigenen Wesen schöpft. So entsteht der Begriff Autonomie, „das Prinzip des durch alle scine Maximen allgemeingesetzgebendon Willens ${ }^{(s 1)}$. Als autonomer Wille aber ist der Wille frei in positivem Sinne. Er ist nicht nur unabhängig von allon empirischen Bestimmungsgründen, frei von sinnlicher Kausalität, sondern selbstthätig frei, indem er sein Verhalten selbstthätig bestimmt und in dem . selbsterzeugten Gesetze mit sich selbst übereinstimmt. „Diese eigene Gesetzgebung der reinen, und als solche, praktischen Vernunft ist Freibeit in positivem Verstande. Also drückt das moralische Gesetz nichts anderes aus, als die Autonomie der reinen praktischen Vernunft, d. i. der Freiheit, und diese ist selbst die formale Bedingung aller Maximen, unter der sie allein mit dem obersten praktischen Gesetz zusammenstimmen können " ${ }^{52}$ ).

6. So ergibt sich mit dem Faktum des Sittengesetzes zugleich die Autonomie der reinen praktischen Vernunft. Es weist damit hin auf eine andere Welt intelligibler Ordnung, das mit ihm steht und fällt. Es ist eine Verstandeswelt, in der eine andere Ordnung der Dinge herrscht, in der die Kausalität der Natur keinen Einfluss mehr hat, die über eine andere Kausalität und andere Gesetze verfügt, nämlich auf Kausalität durch Freiheit.

Es tritt somit neben die Natur unter Gesetze des sinnlichen Mechanismus eine andere intelligible Natur mit eigenen, gänzlich von denen der ersteren verschiedenen Gesetzen. Eine geistige übersinnliche Wirklichkeit thut sich anf, und den Blick in diese andere Welt eröffnet das Bewusstsein des Sittengesetzes. Es ist "die Natur unter der Autonomie der reinen praktischen Vernunft". „Das moralische Gesetz gibt, wenn gleich keine Aussicht, dennoch ein schlechterdings.... unerklärliches Faktum an die Hand, das auf reine Verstandeswelt Anzeige gibt, ja diese sogar positiv bestimmt und uns etwas von ihr, nämlich ein Gesetz erkennen lässt. Dieses Gesetz soll der Sinnenwelt, als einer sinnlichen Natur, die Form einer Verstandeswelt, d. i. einer übersinnlichen Natur

51) Rosenkranz VIII, 62.

5?2) Ebenda VIII, 145 . 
verschaffen, ohne doch jener ihrem Mechanismus Abbruch zu thun $^{\text {(33). }}$.

7. Wie steht es nun mit der Freiheit? Transcendentale Freiheit konnte die theoretische Vernunft nicht aus dem Reich der Möglichkeit verweisen, sie. musste zugeben, dass Kausalität nach Naturgesetzen und Freiheit in der Transcendentalphilosophie keine Widersprüche enthalten, sofern sio notwendig in demselben Wesen gedacht werden, weil sonst nicht Grund angegeben werden könnte, warum wir die Vernunft mit einer Ideo belästigen

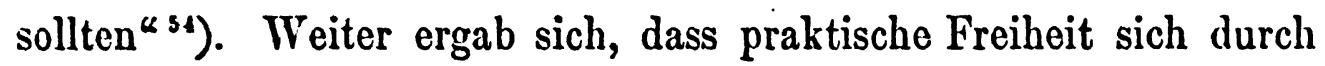
Erfahrung erweisen lasse, nämlich als Unabhängigkeit der Willkür von bestimmenden sinnlichen Notiven. Das aber ist eine Binsenwahrheit. Jenn dass der Mensch durch sinnliche Antricbe, Hang, Neigungen u. dergl. nicht allein „nezessitiert" wird, sondern auch Ueberlegungen und weitgehende Reflexionen sein Thun und Handeln beeinflussen, wenn nicht gar bestimmen, wird niemand bestreiten wollen. Aber die Sache erhält sofort noch eine andere Beleuchtung, wenn man beachtet, wie weit Kant den Begriff Sinnlichkeit ausdehnt. Da wird auch die Wurzel der Ueberlegungen genau geprüft, auch das Erdreich, aus dem sie entsprossen sind, und oft stellt es sich heraus, dass in letzter linie eine niedrige Neigung, ein Hang, ein Lustgefühl, wenn auch feinster Art, die Grundlage war, und so fallt damit auch das ganze nicht ausserhalb des Bereiches des Sinnlichen. Da will denn Unabhängigkeit ron sinnlichen Antrieben recht viel heissen. Es wird damit für den Menschen ein besonderes Gebiet beansprucht, indem er neben dem sinnlichen Notiv auch dem Zuspruch und Urteil der Vernunft sich offen zeigt. So ist in diesem negativen Begriffe der Freiheit schon ein gut Stück der positiven Bedeutung enthalten. Sie ist nicht Willkür in gewöhnlichem Sinne des Wortes aequilibrium, libertas indifferentiae ${ }^{\text {s5 }}$ ), sondern sie ist

s) Elends VIII, $157 \mathrm{f}$.

3) Etenda VIII, 91 f., VIII, 796.

is) Der in der ersten Auflage der Kritik der reinen Vernunft hüufig vorkommende Begrif ist in der zweiten Auflage nacb IJegler (a. a. 0. S. 161 I.) weggelassen, während er in dem grundlegenden Teil der Kritik der praktischen 
nach oiner Bostimmung der 2. Auflage der Kritik der reinen Vernunft: freie Willkür, d. h. eine solche, die „durch Bewegursachen, welcho nur von der Vcrnunft vorgestellt werden, bestimmt werden können"s6). Jene Willkür wäre das Gegenteil und der Tod der Freiheit. Sie ist mithin nicht "gesetzlos", sondern hat auch eine Kausalitït, und zwar eine solche nach "ewigen, unwandelbaren Gesetzen der Vernunft". So ist „Freiheit in positivem Verstande“ „Eigenschaft des Willens sich selbst ein Gesetz zu sein“, d. i. Autonomie, denn "ein freier Wille und ein Wille unter sittlichen Gesetzen sind einerlei ${ }^{\text {(57) }}$ ).

Aus dem Begriff und der Thatsache folgte der Begriff der Autonomie, aus diesem ergab sich der der Freiheit. Es steht und fällt demnach alles mit der Thatsache des Sittengesetzes. Beide, Sittengesetz und Freiheit gehören zusammen, und lassen sich durchaus nicht trennen. Lässt sich das Sittengesetz als objektive Realität beweisen, so erhält damit auch die Freiheit ihre Sanction. Nun wurde oben schon dargethan, dass das Sittengesetz ein Factum, aber gleichsam ein "unerklärliches", wenn auch "unleugbares" sei. Und thatsächlich ist es als Idee ebenso unerklärlich als unvermeidlich. Es ist. Damit ist aber auch die Freiheit. „Sie wird durch das moralische Gesetz und zwar zu dessen Behufe postuliert" $\left.{ }^{58}\right)$. Sie gilt wegen der Realität des Gesetzes als Voraus-

Vernunft sehr zurücktritt. Scharf abgegrenzt ist der Begriff stets gegenüber dem des Begehrungsvermögens; nicht so strenge durchgeführt ist die Scheidung von Willkür und Wille, da der Begriff Wille durçhaus nicht einheitlich gefürbt ist. Beiden ist gemeinsam und charakteristisch gegenüber dem Begehrungsvermögen die Bestimmung des Handelns nach Regeln, wobei für den Willen das Schwergewicht nach der Seite des „von der Vernunft vorgestellten Bestimmungsgrundes“, für die Willkür nach der Seite „der empirischen psychologischen Verwirklichung des Handelns" liegt (Hegler, S. 167). Kant selbst scheidet in der Einl. zur Metaphys. d. S.: „Das Begehrungsvermögen, dessen innerer Bestimmungsgrund, folglich selbst das Belieben, in der Vernunft des Subjekts angetroffen wird, heisst Wille. Der Wille ist also das Begehrungsvermögen nicht sowohl, wie die Willkür, in Beziehung auf die Handlung, als vielmehr auf den Bestimmungsgrund der Willkür zur Handlung betrachtet" (IX, 12).

56) Rosenkranz VIII, 91,

${ }^{57}$ ) Ebenda VIII, 79.

58) Ebenda VIII, 276. 
setzung für alle vernünftigen Wesen. „Nun behaupte ich, dass wir jedem vernünftigen Wesen, das einen Willen hat, notwendig auch die Idee der Freiheit leihen müssen, unter der es allein handelt. Denn in einem solchen Wesen denken wir uns Vernunft, die praktisch ist, d. i. die Kausalität in Ansehung ihrer Objekte hat... Sie muss sich selbst als Urheberin ihrer Prinzipien ansehn, unabhängig von fremden Einflüssen; folglich muss sie als praktische Vernunft... von ihr selbst angesehen werden, d. i. der Wille desselben kann nur unter der Idee der Froiheit ein eigener sein und muss also in praktischer Absicht allen vernünftigen Wesen beigelegt werden ${ }^{(59}$ ). Dadurch soll aber der Kausalität der Erscheinungen durchaus kein Abbruch gethan werden. Die Handlungen müssen erklärt werden nach dem Naturgesetze von Ursache und Wirkung. Sie erscheinen alle natürlich bedingt. Wenn man den Menschen als Phänomenon besser durchschauen könnte, so müsste man sein Leben konstruieren können mit mathematischer Genauigkeit und Richtigkeit, sofern man die äusseren begleitenden Umstände vollständig in Rechnung ziehen könnte. Wenn innerste Triebfedern und äussere Veranlassungen bekannt wären, so könnte man: „eines Menschen Verhalten auf die $\mathrm{Zu}$ kunft mit Gerissheit, sowie eine Mond- oder Sonnenfinsterniss vorausrechnen $\left.{ }^{u 60}\right)$. Das zeigt uns nun auch wie die Freiheit gemeint ist. Kant bezeichnet sie als Idee, und es ist vorauszusetzen, dass unser Philosoph seine eigene, dringende Mahnung in der Kritik der reinen Vernunft, den Begriff der Idee nur in seinem ursprünglichen, reinen Sinne zu brauchen, selbst treulich befolgt hat. Ausdrücklich und an den entscheidenden Stellen immer redet Kant ron der Idee der Freiheit. Sie ,ist nur eine blosse Idec $\left.{ }^{(61}\right)$. „Ein jedes Wesen, das nicht anders als unter der Ideo der Freiheit handeln kann, ist eben darum in praktischer Rücksicht wirklich frei d.i. als gelten für dasselbe alle Gesetze, die mit der Freiheit unzertrennlich verbunden sind, ebenso, als ob sein Wille auch an

\footnotetext{
39) Ebenda VIII, 80 f.

cy Elvenda VIII, 230.

(1) Elenda Vill, 94.
} 
sich selbst und in der theoretischen Philosophie gültig, für frei erklärt würde" 67). Dieses "als ob " ist sehr charakteristisch. Deutlicher kann man den Charakter der Idee als regulative Maxime nicht bezeichnen. So erhält denn die Freiheit als regulative Maxime objektiven Realitätswert. Als solche hat sie grosse Wichtigkeit, wie später gezeigt werden soll. Sie soll nicht die menschlichen Handlungen erklären, sondern sie soll dem Sittengesetz helfen, sich verwirklichen zu können, sie ist kein konstitutives Prinzip für die Erklärung, bezw. für den Beweis der Willensfreiheit, sondern eine regulative Idee, derzufolge wir uns unbeschadet unserer Einbezogenheit in den Zusammenhang der sinnlichen Welt, als Glieder einer intelligiblen Welt betrachten, welche unter dem Gesetze der reinen praktischen Vernunft steht ${ }^{63}$ ).

Die Idee der Freiheit erhebt sich an der Grenze der Erfahrung unvermeidlich. Sie ist die "ratio essendi des Sittengesetzes, wie diese die ratio cognoscendi der Freiheit ist" ${ }^{64}$ ). Dieses ist ein weiter nicht erklärbares Factum. Wer dieses einsieht, über wen sich die siegende Macht desselben als solche erweist, der wird auch die Idee der Freiheit anerkennen müssen. Er wird dann

62) Ebenda VIII, 80.

63) Der Gedanke der Freiheit und seine Darstellung bei Kant begegnet grossen Schwierigkeiten, die sich mehren, je mehr man auf sie eingeht. Auch mit der Betonung der Freiheit als regulative Maxime sind nicht alle Schwierigkeiten gehoben, obwohl sie mir die beste aller Lösungen zu sein scheint, weil sie den in der Kritik der reinen Vernunft entwickelten Grundsätzen Kants am meisten entspricht. Dass Kant immer die Bedeutung der Idee, wie er sie selbst verlangt, reingehalten hat, ist bei dem schwankenden Gebrauch seiner terminologischen Begriffe nicht zu erwarten. Deshalb ist Hegler's Einwand gegen Cohen, der den Begriff der Freiheit als regulative Idee in der schon mehrfach erwähnten Abhandlung eingehend erörtert hat, dass nämlich von Kant "die Freiheit nicht aufgelöst werde in eine regulative Idee", „in einen Gesichtspunkt der Betrachtung“, dass sie vielmehr „eine gesicherte, substantiellere Realität" (Hegler, a. a. 0. 142) habe, nicht ganz unberechtigt, angesichts so mancher Stellen beị Kant, die allerdings den Verdacht aufkommen lassen, dass hier Kant sich selbst nicht treu geblieben sei. - Ob sein ganzer Charakter hier nicht von grossem Einfluss gewesen ist? Die Realität der Ideen Gott, Freiheit, Unsterblichkeit waren ihm persönlich mehr als Ideen, mehr als Postulate der Vernunft.

64) Reicke, a. a. 0. 120. 
nicht mehr fragen, wie Freiheit möglich sei, denn das zu orklären ist gänzlich unmöglich ${ }^{65}$ ).

8. Dieser Gedanke der Freiheit „führt freilich die Idee einer anderen Ordnung und Gesetzgebung als die des Naturmechanismus herbei und macht den Begriff einer intelligiblen Welt notwendig... aber blos ihrer formalen Bedingung nach, d. i. der Allgemeinheit der Maxime des. Willens, als Gesetze, mithin der Autonomie des letzteren, dio allein mit der Freiheit desselben bestehen kann, gemäss zu denken ${ }^{66}$ ). „Durch den unerforschlichen, aber nichts desto weniger unwidersprechlichen Begriff der Freiheit ist sich der Mensch seiner als eines intelligibeln in Ansehung des Naturmechanismus von dieser seinem Einfluss auf seinen Willen unabhängigen Wesens bewusst ${ }^{467}$ ). So verschafft die Freiheit der Idee von einer übersinnlichen Ordnung, an welcher mit Vernunft und Willen begabte Wesen Teil haben, Wirklichkeit.

9. Unabhängigkeit von dem Naturmechanismus war ein Morkmal des Sittengesetzes, das sich schon früher ergab. Hier.folgt aus ihm vermittels der Wechselbegriffe Autonomie und Freiheit die Wirklichkeit einer übersinnlichen Ordnung der Dinge durch das Sittengesetz. Beide Ergebnisse aber bilden die wichtigsten Merkmale des Begriffs der moralischen Persönlichkeit, d. i. „die Freiheit und Unabhängigkeit von dem Mechanismus der ganzen Natur, doch zugleich als ein Vermögen eines Wesens betrachtet, welches eigentümlichen, nämlich von seiner eigenen Vernunft gegebenen, reinen praktischen Gesetzen - die Person also, als zur Sinnenwelt gebörig, ihrer eigenen Persönlichkeit unterworfen ist, soferne sie zugleich zur intelligiblen Welt gehört ${ }^{468}$ ).

10. Somit ergibt sich zunächst: Der Begriff der Persōnlichkeit ist. dio Idee des autonomen vernünftigen Wesens.

Damit ist die Persönlichkeit jenseits aller Erfahrung gestellt

cy) Rosenkranz VIII, 276. 223. 90. 94. 96.

c) Ebenda VIII, 34.

6) Reicke a. 2. 0. 263.

ci) Roseukraz VIII, $214 \mathrm{f}$. 
und bezeichnet das reine, durch kein sinnliches Interesse getrübte Ideal der Menschhoit, deren objektivierten Allgemeinwillen sie in sich begreift. Allein auf Regeln der Vernunft und deren Gesetze gegründet, ist sie allem Wechsel und aller Veränderung entrückt, thronend in ewiger, unwandelbarer Schönheit, wie das Sittengesetz, als dessen Projection sie sich erweist, sie ist das Noumenon des Sittengesetzes. Dieses entspringt der schöpferischen Vernunft. Insoforn ist die Persönlichkeit als das autonome Wesen Subjekt des Sittengesetzes. Da aber das autonome Wesen sich seinem selbsterzeugten Gesetze unterwirft, somit seine eigenen Gesetze auf sich bezieht, so ist die Persönlichkeit zugleich Subjekt und Objekt des Sittengesetzes ${ }^{69}$ ).

B.

Aus dem Begriff dei Persönlichkeit als der Idee des autonomen Wesens und als dem Subjekt des Sittengesetzes ergeben sich noch einige andere wichtige Merkmale für denselben:

1. Autonomie ist nur unter vernünftigen Wesen möglich, da das Sittengesetz nur aus Vernunft entspringt. Dasselbe bezeichnet ferner nur die rein formale Seite der Vernunft, also das was jedem vernünftigen Wesen als solchem zukommt, reine Vernunft. Der Mensch ist ein vernünftiges Wesen. Er hat also Teil an dem Sittengesetz, das sich somit als Produkt eines Allgemeinwillens darstellt. So entsteht die Idee „des Willens eines jeden vernünftigen Wesen als allgemeingesetzgebenden Willens". Dieser Allgemeinwille kann auch gedacht werden als der Wille der ganzen Menschheit. "Somit ergibt sich die Idee der Menschheit als Subjekt des Sittengesetzes. Damit ist eine neue Bestimmung für den Begriff der Persönlichkeit gewonnen: Die Idee der Persönlichkeit ist die Idee der Menschheit "die Idee der Menschheit ganz intellektuell betrachtet" ${ }^{\text {70 }}$ ).

2. Ein vernünftiger Wille kann nur unter Zwecken handeln,

${ }^{69}$ ) In einer Stelle des I. Theils der Religion innerhalb d. Kr. d. r. Vft. identifiziert Kant geradezu Sittengesetz und Persönlichkeit: „Die Idee des moralischen Gesetzes ist die Persönlichkeit selbst" (X, 29).

70) Ebenda X, 29. 
denn nur ein solcher kann Bewegungsgrund des Wollens sein. „Nun ist aber das, was dem Willen zum objektiven Grunde seiner Selbstbestimmung dient, der Zweck, und dieser, wonn er durch blosse Vernunft gegeben wird, muss für alle vernünftigen Wesen gleich gelten." Dann aber ist es ein Zweck an sich, ein Selbstzweck. Einen solchen kann nur der Gesammtwille setzen. Nicht was jeder als $Z$ weck will, kann objektive Geltung beanspruchen, sondern nur der Zweck der Menschheit. Zweck der Menschheit ist aber das Sittengesetz, das somit zum Zweck an sich, zum Endzweck wird. Damit wird aber auch das Subjekt des Sittengesetzes zum Endzweck und es ergibt sich: Die Idee der Persönlichkeit als absoluter Zweck, als Endzwock ").

3. Damit aber erhält die Persönlichkeit einen unendlichen Wert. Alle Zwecke haben einen Wert, auch die subjektiven. Der relative Wert der subjektiven $Z$ wecke verschwindet aber vor dem unendlichen Werte eines Zweckes an sich selbst. Die Persönlichkeit als Subjekt des Sittengesetzes, als das autonome Wesen besitzt eine Würde, einen absoluten Wert, die sie über alles Sinnliche hinaushebt und sie jenseit alles Begehrenswerten stellen. Sie wird zur übersinnlichen Grösse, deren Majestät unbedingte Achtung heischt. Die Idee der Menschheit, oder was dasselbe ist, die Idoc der Persönlichkeit hat den höchsten, erdenkbaren Wert als $\mathrm{Z}$ weck an sich selbst oder als Endzweck. Die Menschheit selbst ist eine Würde; deun der Mensch kann von keinem Nenschen blos als Nittel, sondern muss jederzeit zugleich als Zweck gebraucht werden, und darin besteht eben seine Würde, seine Persōnlichkeit" ${ }^{73}$.

4. Die Persōnlichkeit ist demnach als Subjekt des Sittengesetzes Glied einer durch dasselbe begründeten, unter Gesetzen der

i1) Die Idee der Jenschbeit als Zreck, als Endzweck, ist eine der Lieblingsideen Kants. In der Grundlegung z. M.d.S. benutzt er sie sogar zu eiver zweiten Formulierung des kategorischen Imperativs, die das formale Joralprinzip der ersten Formulierung durch ein materiales (Idee der Jenschheit als Zweck) ersetzt, ja jene sogar ganz verdrängt. Dic Idee der Btenschheit als absoluter Zweck ist in den angeraudien, ethischen Schriften geradezu die Grundlage, aus der er alle Tugenden und PAicbten ableitet.

in) Rosenkranz IX, 325.

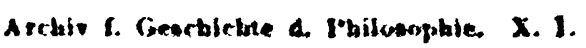


Freiheit stehonden intelligibeln Ordnung. Sie bedeutet die Idee der Menschheit als Noumenon und ist daher Endzweck. Darin beruht ihr absoluter Wert und ihre Erhabenheit.

Sie ist eine Idee. Damit ist deutlich genug gesagt, dass in der Erfahrung eine ihr entsprechende Anschaung nicht gefunden werden kann. Die Vernunft ist wohl imstande sich diese Idee zu schaffen, ihrem Willen dieses Ideal vorzustellen, aber es fehlt ihr das physische Vermögen, diesem Ideal Verwirklichung zu verschaffen. Der Wille ist der natürlichen Ordnung, dem Naturgesetze unterworfen, die keinen Eingriff in ihr Gebiet verstatten. Bleibt somit, fragt man sich, die Idee der Persönlichkeit ein blosses Gedankending und deshalb völlig bedeutungslos? Oder hat sie neben dem idealen auch realen Wert?

C.

1. Die Beantwortung dieser Frage, die uns zum Schlusse noch beschäftigen soll, ist eigentlich schon gegeben mit der Betonung des Begriffs der Persönlichkeit als Idee. Damit ist gesagt, dass er nur als regulative Maxime praktischen Wert hat. Und gerade hierin ruht die Erhabenheit dieser Idee. Das Ideal, das als solches allgemein und unter allen Umständen gelten soll, darf mit der Bedingtheit durch Erfahrung nichts zu thun haben. Es soll weder der Psychologie, noch der Anthropologie entnommen sein; wenn es Anspruch auf objektive Realität machen will. Gerade dass es jenseit, an der Grenze aller Erfahrung als regulative Maxime sich erhebt, macht seinen eminenten Wert aus.

Wie kann aber diese Idee praktisch wirken, da doch der Mensch mitsamt seinem Willen der Bestimmtheit durch die Naturgesetze unterworfen ist? Auch diese Frage löst die Bedeutung der Idee als regulative Maxime.

2. Nicht in geheimnisvoller, rätselhafter Weise wirkt die intelligible Persönlichkeit auf die empirische ein. Nicht in diesem Sinne ist ihre Wirksamkeit aufzufassen. Ihre Wirkung ist eine passive und führt absolut nichts Geheimnisvolles, Mystisches mit sich.

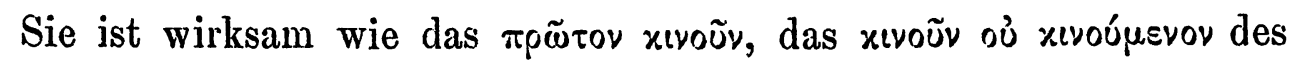
Aristoteles, das in ewiger, selbstgenügender Schönheit jenseits alles 
Wechsels und aller Veränderung thront; ohne zu wollen zieht es Alles durch seine bezaubernde Harmonie an sich und nach sich: Das Geheimnis liegt in der Bedeutung der Idee als Zweckidee. Als regulative Naxime will sie sagen: An der Idee der Persönlichkeit soll das vernünftige Wesen seine Handlungen nach ihrem sittlichen Wert bemessen, sie soll die Norm für Bestimmung des Willens sein, gleich als ob er auch gemäss der Regel dieser Idee handeln könne.

Es wird mit dieser Idee nicht ein übersinnliches mystisches Selbst statuiert. Es ist das idealo Subjekt des Menschen, das seine Vernunft mit unvermeidlicher Sicherheit sich schafft, das sein besseres Ich ausmacht. Man darf es nicht versinnlichen, man soll sich ron ihm kein Bildnis machen. Als regulative Maxime erzeigt sich die Idee als äussorst wertvoll, indem sie reinigend und klärend, mahnend und erziehend thätig ist, das Urteil über das, was gut und böse ist, aufhellt und schärft, und dem Menschen das Ideal seiner Bestimmung als leuchtendes Beispiel vor das innere Auge hiilt. Das Bewusstsein dieser Idee ist in jedem, auch dem gemeinsten Menschenverstande vorhanden und erzeigt sich dort als bedeutsamer Faktor. Dass diese Idee wirklich unseren Willensbestimmungen gleichsam als Vorzeichnung zum Muster liege, bestätigt die gemeinste Aufmerksamkeit auf sich selbst ${ }^{\alpha 18}$ ). Kant führt zum Beleg dieser Thatsache manigfache Beispiele vor. Das vernichtende Erteil des Gewissens: so handelt kein Mann, der aul diesen Ehrennamen Anspruch macht, beweist seine mächtige Kraft tiiglich und legt so Zeugnis ab für die Wichtigkeit der regulativen Wirkung der Mlaxime, die thätig ist, ,indem es auf die Sittlichkeit des Subjekts Einfluss hat und ein Gefühl bewirkt, welches dem Einfluss des Gesetzes auf den Willen beförderlich ist" ").

3. Dies Gefühl ist das Gefühl der $\Lambda$ chtung, durch welches sich ${ }_{n}$ das intelligible Selbst ${ }^{*}$ als ${ }_{n}$ nötigend in Ansehung des Selbst der Erfahrung beweist". Staunen und Bewunderung erfasst den Nenschen beim Anblick dieser Idee und nötigt ihn Achtung ab,

is) Elienda VIII, liss.

if Ebends VIII, 200. 
„da cs denn nicht zu verwundern ist, wenn der Mensch als zu beiden Welten gehörig sein eigenes Wesen in Beziehung auf seine zweite und höchṣte Bestimmung, nicht anders als mit Verehrung, und die Gesetze derselben mit der höchsten Achtung betrachten muss" ${ }^{75}$ ). Diese Achtung ist kein psychologisches Gefühl der Lust oder Unlust, sie ist einfach der Tribut, den der Mensch dieserer Erhabenhoit zollen muss.

Diese „Achtung erweckende Idee der Persönlichkeit, welche uns die Erhabenheit unsrer Natur (ibrer Bestimmung nach) vor Augen stellt, indem sie uns zugleich den Mangel der Angemessenheit unsres Verhaltens in Ansehung desselben bemerken lässt und dadurch den Eigendünkel niederschlägt ${ }^{\text {( } 76}$ ), erweist sioh gerade durch diese Erzeugung der Achtung als wirksam. Aus der Achtung, die zugleich den Menschen über sich selbst erhebt und ihn demütigt, entspringt die Nötigung des Willens, gemäss dieser Idee zu handeln aus Achtung vor der Erhabenheit dieser Idee, das Sollen, die Pflicht. Sie fasst nichts in sich „was Einschmeichelung bei sich führt", sondern verlangt Unterwerfung und zwar unbe-. dingte ohne Rücksicht auf Hang und Neigungen, die vor ihr "verstummen" müssen, die aber auch nicht durch Zwang sich Eingang verschafft, sondern einfach die Idee in ihrer überwältigenden Macht vor Augen stellt und sich so selbst Verehrung, wenn auch wider Willen schafft. Sie allein kann dem Menschen einen Wert geben. Die "Wurzel ihrer edlen Abkunft, welche alle Verwandtschaft mit Neigungen stolz ausschlägt, kann nicht Minderes sein, als was den Menschen über sich selbst erhebt, die Persönlichkeit" ${ }^{47}$ ).

75) Ebenda VIII, 215.

76) Ebenda VIII. 215.

${ }^{77)}$ Ebenda VIII. 215. In der Religion i. d. G. d. r. V. redet Kant von der Anlage zur Persönlichkeit, die er geracle in der Empfänglichkeit der Achtung vor dem Sittengesetz und deren Wirksamkeit als Triebfeder sieht. „Die Anlage für die Persönlichkeit ist die Empfänglichkeit der Achtung für das moralische Gesetz, als einer für sich hinreichenden Triebfeder derWilikür . . . dass wir diese Achtung zur Triebfeder in unsere Maximen aufnehmen, der subjektive Grund hierzu scheint ein Zusatz zur Persönlichkeit zu sein, und daher den Namen einer Anlage zum Behuf desselben zu verdienen." (Erstes Stück X, 29f.) Die Wurzel dieser Anlage liegt in der unbedingt gesetzgebenden Vernunft. 
3. Der Mensch ist Persönlichkeit "seiner Bestimmung" nach. Als vernünftiges Wesen, das fähig ist, den Gedanken des Sittengesetzes zu erfassen, nimmt er Teil an jener höheren Wirklichkeit, deren Bürger die Persönlichkeit ist. Es kommen ihm deshalb schon jetzt die wesentlichen Eigenschaften und Merkmale des transcendentalen Begriffes zu. Er ist frei und autonom, er soll stets so handeln und seine Handlungen so beurteilen, als ob er frei wäre, d. h. fähig, unabhängig von allen sinnlichen Motiven, nur nach Regeln der Vernunft scino Willensbestimmungen zu treffen.

4. Als vernünftiges Wesen ist ferner die Person, die sich der Persönlichkeit unterwerfen soll, ist der Mensch, wie die Persöulichkeit Zweck an sich, der niemals als Mittel gebraucht werden darf. Als solchen hat jeder Nensch sich selbst und andere zu betrachten. Keiner darf sich jemals sich seiner selbst oder eines anderen als Nittel bedienen oder auch nur als solches anselın, was der Ileiligkeit der Persönlichkeit als der Idee der Menschheit vollständig widersprechen würde. Unter diesem Gesetze stehen alle

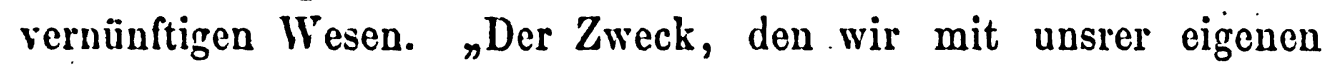
Person haben sollen, ist negativ: die Menschheit in uns will nicht, dass wir den Jenschen zum Nittel erniedrigen sollen" ${ }^{78}$ ). Aus diesem nallgemeinen Zweckvorzug vernünftiger Wesen, erwächst nun die Idee eines gemeinsamen $Z$ weckes aller vernünftiger Wesen, eines "Ganzen“, eines Reiches aller Zwecke". Durch das Gesetz, unter dem sie alle stehen, dass jeder sich selbst und Andere stets als Zwecke an sich selbst betrachten solle, ${ }_{n}$ entspringt eino systematische Verknüpfung vernünftiger Wesen durch gemeinschaftliche objektive Gesetze, d. i. ein Reich, welches, weil diese Gesetze eben die Beziehung dieser Wesen auf einander als $Z$ wecke und Mittel zur Absicht haben, ein Reich der Zwecke heissen kann" ${ }^{19}$ ). $\mathrm{Zu}$ diesem Reich, $n$ dem herrlichen Ideal eines allgemeinen Reiches der Zwecke an sich selbst", gehört die Person. Aus diesem Grund muss uns die Yenschheit in unsrer Person heilig sein ${ }^{2}$ weil er Subjekt des moralischen Gesetzes, mithin dessen ist, was an sich

in Ebenda VIII, 63.

if Reicke, a. a. 0. II, 315. 
heilig ist, um dessen Willon und in Einstimmung mit welchem auch überhaupt nur etwas heilig genannt werden $\mathbf{k a n n}^{\ll 80}$ ).

So ist dic Persönlichkeit das, „was den Menschen über sich selbst als einen Teil der Sinnenwelt erhebt, was ihn an eine Ordnung der Dinge knüpft, die nur der Verstand denken kann, und die zugleich die ganze Sinnenwelt mit. ihr das empirisch bestimmbare Dasein des Menschen in der Zeit und das Ganze aller Zwecke unter sich hat" ${ }^{81}$ ).

5. Sie ist Ziel und Zweck des Menschen. Ihr soll er seine eigene Person unterwerfen, damit er zur Persönlichkeit werde. Dieses Soll gebietet ihm die Pflicht in deutlicher nicht misszuverstehender Sprache. Diesem "du sollst", dem Pflichtgebot aber stellen sich Meinungen, Triebe, Hang, kurz das "pathologisch bestimmbare Selbst" entgegen. Es entspinnt sich so in dem Menschen, der seinem Ziele zustrebt ein Kampf zwischen Pflicht und Neigung, indem die regulative Idee stets Regeln des Verhaltens gibt, mahnt und tadelt. Niemals gelangt der Mensch dazu sein Ideal zu verwirklichen, weil er an die Sinnlichkeit gebunden ist, und niemand, so lange er atmet. aus seiner Haut fahren kann. So ist die Persönlichkeit stets nur im Werden begriffen. Sie erhebt sich von dem Niveau des Sinnlichen, wie der Dampf des Wassers aufsteigt in die Lüfte. Weit und hoch mag er sich erheben, aber niemals kann und darf er die Erde verlassen, stets bleibt er gebunden an sie, die ihre Kinder eifersüchtig bei sich festhält. Wohl kann der Mensch seiner Triebe und Neigungen fast völlig Herr werden, aber niemals kann er sie töten. Es ist ein Kampf mit der Hydra, an Stelle eines abgeschlagenen Hauptes, wachsen womöglich deren zwei.

6. Eine völlige Uebereinstimmung des Willens mit dem Sittengesetze, Vollkommenheit erlangt also der Mensch nie. Kant nennt diese "völlige Angemessenheit des Willens zum moralischen Gesetz" "Heiligkeit". Sie kommt nur der höchsten Intelligenz, dem unendlichen Wesen zu. Ihrer ist „kein vernünftiges Wesen der Sinnenwelt in keinem Zeitpunkt seines Daseins fähig “s2). Indes

80) Rosenkranz VIII, $273 \mathrm{ff}$.

81) Ebenda VIII, 214.

s2) Ebenda VIII, 261. 
würde es, falls keine Aussicht wäre auf eine derartige Vollendung, wertlos sein, dem Ideal nachzustreben. Es hätte keinen Sinn, um eine blosse Idee erfolglos sich zu bewerben und alles andre, Glückseligkeit, um ihretwillen auszuschlagen. Die Vernunft fordert dio Möglichkeit dieser Vollendung. Kann sie innerhalb der Sinnenwelt in der Zeit nicht erreicht werden, so doch in der Unendlichkeit. „Da sie (die Vollkommenheit) indessen gleichwohl gefordert wird, so kann sie nur in einem ins Unendliche gehenden Progressus zu jener völligen Angemessenheit angetroffen werden und es ist nach Prinzipien der reinen praktischen Vernunft, notwendig, eine solche praktische Fortschreitung als das reale Objekt unsres Willens anzunchmen. Dieser unendliche Progressus aber ist nur unter Voraussetzung einer ins Unendliche fortdauernden Existenz und Persönlichkeit desselben vernünftigen Wesens (welche man dio Unsterblichkeit der Seele nennt) möglich ${ }^{483}$ ).

Somit erhalten wir als Resultat unsrer Untersuchung:

Grundlage und Voraussetzung der moralischen Persönlichkeit ist die empirische Persönlichkeit, das mit Verstand begabte Wesen, welches in der auf Denken beruhenden Vorstellung des Ich eine numerisch-identische Einheit für die Manigfaltigkeit der Anschaung und der Vorstellungen hat. Sie ist eine Erfahrungsrealitat.

Die moralische Persönlichkeit dagegen ist eino Idec. Sic bezeichnet das noumenale Subjekt des Sittengesetzes, das den Allgemeinwillen vernünftiger Wesen, somit auch der Menschheit ausdrückt. Wesentlichste Merkmale sind Freihcit und Unabhängigkeit von dem Naturmechanismus und Zugehörigkeit $z u$ einer intelligibeln Ordnung der Dinge, zu einem Reich der $Z$ wecke. Sie ist Endzweck und hat daher absoluten Wert. Insofern sie $Z$ weckideo ist, erhält sie praktische Bedeutung als regulative Maximo durch das Pflichtgebot.

Indem der Mensch fihig ist, den Gedanken des Sittengesetzes zu fassen, in welchem er wich ein Ideal schafft, das jenseit aller

13) Ebenda VIll, 261 a. 
empirischon Bedingtheit steht; jndem or sich die ihm entsprechende Idee der Persönlichkeit zur Maxime seines Verhaltens macht; zeigt er sich als Wesen, das berufen und wärdig ist, auch dieses Ideal zu verwirklichen und einer höheren geistigen Wirklichkeit anzugehören. Der Verstand, die Vorstellung des Ich, hoben ihn unter der Sinnenwelt auf die höchste Stufe, gaben ihm eine Sonderstellung vor den Tieren, aber vermochten nicht, ihn in irgend einer Weise an irgend einer höheren Wirklichkeit der Dinge teilnehmen $z u$ lassen. Teilhaftig wird er derselben als Noumenon und zwar durch die Idee des Sittengesetzes und des Subjekts desselben, der Persönlichkeit. Je mehr er lernt diese Idee zur regulativen Maxime seines Verhaltens zu machen, um so mehr nähert er sich ihrer Verwirklichung, einer geistigen Wirklichkeit, einer Welt der Vernunft, einem Reiche der Zwecke. Diese Idee ist eine Schöpfung der Vernunft; auf ihr, der praktischen Vernunft beruht im letzten Grunde die Persönlichkeit. Die Vernunft macht aus dem „Tiermenschen", dem Verstandesmenschen den Vernunftmenschen; aus der Person die Persönlichkeit.

Das ist des Menschen Bestimmung. Das ist sein Stolz, seine Freude. „Zwei Dinge erfüllen das Gemüt mit immer neuer Bewunderung und Ehrfurcht, je öfter und anhaltender das Nachdenken sich damit beschäftigt: der bestirnte Himmel über mir und das moralische Gesetz in mir . . . . das zweite fängt von meinem unsichtbaren Selbst, meiner Persönlichkeit an, und erhëbt meinen Wert als Intelligenz unendlich durch meine Persönlichkeit, in welcher das moralische Gesetz mir ein von der Tierheit und selbst von der ganzen Sinnenwelt unabhängiges Leben offenbart, wenigstens, soviel sich aus der zweckmässigen Bestimmung meines Daseins . . . . abnehmen lässt" ${ }^{84}$ )

Diese Bestimmung des Menschen zur Persönlichkeit ist Ziel aller Erziehung. Die Idee der Persönlichkeit, dieses erhabenen Zwecks des Menschen soll geweckt und gepflegt werden, und so die Menschheit immer näher der Verwirklichung ihres Ideals entgegengeführt werden. Kant glaubte enthusiastisch an den sittlichen

8)) Ebenda VIII, 312. 
Fortschritt des Menschengeschlechts. „Es ist ein entzückender Gedanke sich vorzustellen, dass die menschliche Natur immer besser werde durch Erziehung entwickelt werden . . . . dies eröffnet uns den Prospekt zu einem künftigen glücklichen MIenschengeschlechte ${ }^{485}$ ). Dies geschicht langsam, stetig, sicher. Es handelt sich nicht darum, ${ }_{n}$ durch Circens Zauberrute auf einmal Vieh in Menschen verwandeln wollen ${ }^{48}$ ), sondern die Umwandelung kann sich nur sicher erzielen lassen auf dem Wege der Erziehung; diese Aufgabe hat die moralisch praktische Erziehung und „sie ist Erziehung zur Persönlichkeit ${ }^{\text {(87)}}$ ).

\section{III.}

Schlussbemerkungen.

Resultat unsres 1. Teils war: der Verstand als das Vermögen der Synthesis des Manigfaltigen macht das Wesen der empirischen Persönlichkeit aus. Er bringt das allgemeine Bewusstsein zustande, indem er die Vorstellungen nach einheitlichen Regeln untereinander verbindet. Dieses allgemeine Bewusstsein hat zur Voraussetzung die transcendentale Apperception, das reine Selbstbewusstsein, ohne das es überhaupt kein Bewusstsein geben kann. Somit ist die transcendentale Persönlichkeit Voraussetzung der empirischen. Als solche sieht sich der Mensch hineingestellt in eine Welt der Notwendigkeit. Er beurteilt alle seine Handlungen nach dem Gcsetz der Causalität von Ursache und Wirkung.

Dic moralische Persönlichkeit dagegen schafft sich innerhalb dieser Welt eine neue, eine Welt der Freiheit, nicht der regellosen Willkür, sondern auch Gesetzen unterstehend, aber selbstgeschaffenen der Freiheit. Als Bürger dieser intelligibeln Welt beurteilt die vernünftige Persönlichkeit alle ibre llandlungen nach dem Gesetz der Freiheit, betrachtet sich als absoluten $Z$ weck und gewinnt damit eine über alles erhabene Würde. So ergab der zweite Teil.

8) Ehenda 1X, 373.

of Reicke 2. 2. 0. II, 87.

i) Rosenkranz IX, 386 . 
Vorstandes- und Vernunftpersönlichkeit bezeichnen somit zwei grundverschiedene Seiten eines und desselben Wesens. Sonst haben sio nichts Gemeinsames. Was berechtigt nun Kant für beide so seh. vorschiedenen Erscheinungsweisen dieselbe Bezeichnung, nämlich Persönlichkeit zu. brauchen?

Kant hat sich die Frage nach der Beziehung zwischen .empirischel und moralischer Persönlichkeit nirgends ausdrücklich gestellt, auch nirgends angedeutet. Doch ist es nicht schwer aus dem Ganzen seiner Untersuchungen heraus diese Frage ganz in scinem Sinne $z u$ beantworten.

2. Kant führt den Begriff der transcendentalen (empirischen) Persönlichkeit ein, veranlasst durch die kritische Erörterung der metaphysischen Ansicht von der Seele. Somit ist der Begriff eigentlich nur gelegentlich gebraucht und spielt keine grosse Rolle. Weil Kant ihn vorfand, liess er ihn gelten, nachdem er ihm jede metaphysische Färbung genommen. „Indessen kann, so wie der Begriff der Substanz und des Einfachen ebenso auch der Begriff der Persönlichkeit (sofern er blos transcendental ist) . . . bleiben" (II, 292). Er begegnet erst wieder von da ab in der Kritik der praktischen Vernunft und den übrigen praktischen Schriften unter gänzlich veränderter Bedeutung. Und doch stehen beide in inniger Verbindung.

In einer Anmerkung der 2. Auflage der Kritik der r. Vernunft bemerkt Kant einmal „die synthetische Einheit der Apperception ist der höchste Punkt, an dem man allen Verstandesgebrauch, selbst die ganze Logik und die ganze Transcendentalphilosophie heften muss" $\left.{ }^{88}\right)$. Er hätte noch hinzufïgen können: auch die ganze praktische Philosophie. Denn ohne Beziehung auf transcendentale Apperception ist keine Erkenntnis möglich. Alle Vorstellungen erhalten erst Realität durch die Beziehung auf das "Ich denke“. Auch die unter der Idee der Freiheit sich ergebenden Vorstellungen müssen auf das Selbstbewusstsein bezogen werden, sonst bleiben sie unmöglich oder tot. So ist denn ohne die transcendentale Apperception, die das Wesen der Verstandespersönlichkeit aus-

ss) Ebenda II, 733. 
macht, die moralische Persönlichkeit mit ihren sittlichen Vorstellungen nicht denkbar oder mit anderen Worten: die Verstandespersönlichkeit ist Grundlage und Voraussetzung dêr Vernuuftpersönlichkeit.

Auch von einem andern Punkt aus gelangt man zu diesem Resultat. Das Sittengesetz soll sich wirksam erzeigen. Da es Regeln vorschreibt über das, was geschehen soll, so kann es seine Wirksamkeit nur entfalten in der Anwendung der Regeln auf Handlungen. Letztere aber fallen zweifellos in das Gebiet der Erfahrung. Diese nun ist, wie schon mehrfach betont, gar nicht möglich ohne die synthetische Arbeit des Verstandes. Das Sittengesetz bedarf um wirksam werden zu können der Erfalurung, die wiederum die Beziehung auf das "Ich denke" erfordert oder die moralische Persönlichkeit setzt die empirische als Grundlage voraus.

3. Die Freiheitspersönlichkeit ist, wio oben gezeigt wurde, der absolute Endzweck der Menschheit. Diese kann sich aber nur in der Auseinandersetzung mit der Sinnlichkeit und ihrer Ceberwindung entwickeln und gestalten. Sio muss sich innerhalb der Erfahrung bethätigen, dicselbe sich ihr dienstbar machen. Ylittel ist die empirische Person. So ist die empirische Persönlichkeit nicht nur conditio sine qua non der moralischen, sondern auch Vittel für ihre Verwirklichung. Diese Gedauken führen direkt zu Fichte.

4. Beide angeführte Beziehungen sind von Kant nirgends ausdrücklich berührt. Er hat vielmehr nie versucht, Vernunft und transcendentale Apperception in Verbindung zu bringen und so ein System der Vernunft zu schaffen, obwohl er an letzteres dachte. Er fragt auch nicht nach dem Wesen der Apperception. Beide, Vernunft und Selbstbewusstsein gehen unvermittelt nebeneinander her, obwohl es nahe lag, in letzterem den gesuchten gemeinsamen Grund für alle „Vermögen“ zu schen.

5. Schroffer wird der Gegensatz zwischen der empirischen und moralischen Persönlichkeit in der Entgegensetzung von Vernunft und Sinnlichkeit. An die Vöglichkeit ihrer Einheit dachto Kant allerdings, wie eine Stelle der Einleitung zur Kritik der reinen V'crnunft (1. Auflage) zeigt. „Nur sovicl scheint zur Ein- 
leitung ... nötig zu soin, dass es zwei Stämme der menschlichen Erlicuntnis gebe, dio vielleicht aus einer gemeinschaftlichen, aber uns unbekaunten Wurzel entspringen, nämlich Sinnlichkeit und $V^{\prime}$ crnunft $\left.{ }^{(89}\right)$. Diesen Gedanken, nach dieser gemeinsamen Wurzel zu forschen und damit seinem System eine geschlossene Einheit zu geben, verlor er immer mehr aus dem Auge, je mehr er sich mit der praktischen Philosophie beschäftigte. Zu einer Ausführung kam es nie. Nicht nur weist er die Sinnlichkeit völlig aus der Begründung des Sittengesetzes, diese tritt sogar als Feindin der letzteren auf, die deren Verwirklichung überall in den Weg tritt, so dass man sich oft gerne dieser Fessel entledigt sähe. So ergibt sich denn die ungeheure Kluft im Wesen des Menschen, ein unerträglicher Dualismus zwischen Sinnlichkeit und Vernunft, der nur gar zu sehr an den von Kant zerstörten Dualismus von Leib und Seele erinnert. Es lag eigentlich sehr nahe; den Gedanken der transcendentalen Apperception weiter zu verfolgen und in ihr die gemeinsame Wurzel der beiden diametral einander entgegenstehenden Stämme der menschlichen Erkenntnis zu finden, da ja beide ohne Selbstbewusstsein nicht sein können. Aehnliches ist es mit dem Verhältnis der theoretischen und praktischen Vernunft. Auch hier ist Kant über Ansätze und Andeutungen nicht hinausgekommen. El setzt ihre Einheit gleichsam voraus, obne näher auf sie einzugehn. Als nachher Fichte bei der transcendentalen Apperception einsetzte, um diese Lücke des Idealismus zu schliessen, protestierte Kant sehr lebliaft dagegen, ein Beweis, wie sehr obiger Gedanke bei ihm in Vergessenheit geraten war ${ }^{90}$ ).

Die ganze Grösse der Schwierigkeit, in die sich Kant hier setzt, zeigt sich erst, als er sich anschickt, die Einwirkung des der Vernunft entsprungenen Sittengesetzes auf das sinnlich bedingte Ich zu erklären. Hier wird es ihm sehr schwer, nachdem er vorher in der Begründung des Sittengesetzes alle Brücken zwischen der Vernunft und der Sinnlichkeit abgebrochen hat, wieder eine Verbindung anzuknüpfen. Als der vermittelnde Begriff erscheint hier die Achtung, wobei sich aber so recht die

89) Ebenda II, 28.

${ }^{90)}$ cf. Hegler, a. a. 0. $113 \mathrm{ff}$. 
Verlegenheit zeigt, die unser Philosoph sich selbst bereitet hat. Einerseits soll sie ein Gefühl sein, andrerseits soll sie kein Gèfühl sein. Kant fühlt das Unbehagliche der Lage selber, und es gelingt ihm auch nicht, hier lichtvolle Klarheit zu schaffen.

6. Wir sehen, dass es -ganz in der Konsequenz der Kant'schen Ausführungen liegt, wenn man die empirische Persönlichkeit als Grundlage der moralischen und als Mittel zu ihrer Verwirklichung bezeichnet. Ferner, dass der einzig mögliche Weg zur Beseitigung des Dualismus von Vernunft und Sinnlichkeit, ohne von Kant abzuweichen, nur in der Zurückführung beider auf dio transcendentale Apperception liegen kann. Fichte schlug beide Wege ein mit dem vollen Bewusstsein nur im Sinne der Transcendentalphilosophie weiter zu bauen:

Er stellt sich wie auch Kant die Frage nach dem Grund aller Erfalirung. Aber Kant bleibt bei dem a priori stehen, während Fichte auch dessen Grundlage zu erforschen sucht. Er geht von der Thatsache aus, dass im Bewusstsein sich Vorstellungen finden von zweierlei Art, solche, „die von dem Gefühl der Notwendigkeit begleitet $\operatorname{sind}^{\text {(91) }}$ ) und willkürliche. „Welches ist nun der Grund", so fragt er sich, „des Systems dieser Vorstellungen und dieses Gefühls der Notwendigkeit selbst?" oder „wie kommen wir dazu, dem das doch subjektiv ist, objektive Notwendigkeit beizumessen, oder ein Sein anzunehmen?( ${ }^{92}$ ). Objektives Sein gibt es nur für ein Subjekt, sein Grund kann also nur im Subjekt liegen. Da aber Grund des Seins nicht wieder ein Sein sein kann, so bleibt in dem Subjekt, welches réin von aller Vorstellung des Seins ist, nichts mehr übrig als ein Handeln, das sich nur auf sich selbst bezichen kann und in sich selbst zurückgeht, sich selbst anschaut. Diese ,intellektuelle Anschauung " bezeichnet Fichte als das Selbstbewusstsein, das Ich, das reine Ich.

Sie ist genau dasselbe, was Kant mit transcendentaler Apperception bezeichnet. Kant nennt diese einen $\Lambda$ ctus der Spontaneität, der alles Jenken und Vorstellen erst möglich macht, Fichte ein Ilandeln, eine Thathandlung des reinen Ich, das lediglich durch sich

9) Fichte, Werke. Berlin 1845, Bd. I, 423.

ग) a. 2. 0.456. 
sellust alsolut bestimmt ist", durch die das reine Ich sich seiner erst bewusst wirl. Dic intollektuelle Anschaung ist „die Quelle alles Lebens, ohne sie ist der Tod ${ }^{43}$ ). Man sieht, wie hier Fichte ganz mit Kant sich berührt, nur klarer, bestimmter, schärfer ist scinc "intellektuelle Anschauung". Diese Thathandlung ist grundlos, ein Akt der Freiheit, sie ist Anfang und Ursache alles Seins und Handelns. Das Ich beschränkt sich selbst durch Setzung des Nicht-Ich, so entstehen die Vorstellungen, die Deukgesetze, die Kategorien, die Welt der Erfahrung, indem das Ich immer wieder über die gesetzte Schranke hinausgeht, denn sein Streben ist unendlich, es ist im Grunde praktisch. Um unendlich sein zu können, schaftt sich das Ich immer neue Schranken, neue Widerstände seiner Thätigkeit; denn mit dem Aufhören der Widerstände würde auch seine Thätigkeit aufhören. So wird das theoretische Ich Mittel und Grundlage zur Bethätigung des praktischen Ich. Dieses ist nicht nur Grund, sondern auch Zweck des Strebens. So ist „der Begriff des Handelns, der nur durch diese intellektuelle Anschauung des selbstthätigen Ich möglich wird, der einzige, der beiden $W$ elten, die für uns da sind, vereinigt, die sinnliche und die intelligible. Was meinem Handeln entgegensteht - etwas cntgegensetzen muss ich ihm, denn ich bin unendlich - ist die sinnliche, was durch mein Handeln entstehen soll, ist die intelligible Welt" $\left.{ }^{94}\right)$. Ziel der Welt und alles Seins ist Realisierung des Sittlichen, des praktischen Ich, Grund das unendliche Streben des Ich, beginnend in dem Moment, wo der Mensch zum ersten Mal Ich denkt (man vergleiche hierzu Kant's Einleitung in seine Anthropologie), Mittel die vom Ich erzeugte. Welt der Vorstellung, die Erfahrung. Setzt man für das Ich einmal transcendentale und dann moralische Persönlichkeit, so hat man ganz die Gedanken Kant's, nur eben in andrer Form und reicherer Ausführung. So ist Fichte der Fortsetzer der Gedanken Kant's.

7. Dies ergibt sich auch noch in einem anderen Punkte. Die empirische Persönlichkeit, soferne sie zugleich transcendental ist (cf. I, 3), weist als Erscheinung (phaenomenon) hin auf ein Ding

93) a. a. 0.463.

94) a. a. 0.467. 
an sich, auf die Persönlichkeit als Noumenon. Noumena. bleiben der theoretischen Vernunft ihrem Wesen nach unbekannte Dinge, über die sio weiter nichts sagen kann, als dass sie dieselben denken muss. So bleibt auch das Noumenon der Persönlichkeit gänzlich unbekannt, da ihm in der Erfahrung kein entsprechender Gegenstand gegeben werden kann. Diese leere Stelle füllt die Lehre von der Freiheit aus. Das Noumenon der empirischen Persönlichkeit ist die moralische. Alles, was die theoretische Vernunft leisten konnte, war ;den Gedanken von einer freihandelnden Ursache, wenn wir diese auf ein Wesen in der Sinnenwelt, sofern es andrerseits auch als Noumenon betrachtet wird, anwenden $z u$ verteidigen ${ }^{(95}$ ). „Ich konnte aber diesen Gedanken nicht realisieren, d. i. nicht in Erkenntnis eines so handelnden Wesens, auch nur blos seiner Möglichkeit nach, verwandeln. Diesen leeren Platz füllt nun reine praktische Vernunft, durch ein bestimmtes Gesetz der Kausalität in einer intelligiblen Welt (durch Freiheit) nämlich das moralische Gesetz aus $\left.{ }^{46}\right)$. So wird der Mensch als moralisches zur Kehrseite des empirischen Wesens, die moralische Person zum Ding an sich, denn der Mensch fühlt sich als Persönlichkeit, als cinheitliches Wesen, „das als zur Sinnenwelt gehörig, sich gleich anderen wirksamen Ursachen, notwendig den Gesetzen der Kausalität unterworfen erkannt, im Praktischen, doch zugleich sich auf einer andern Seite, nämlich als Wesen an sich selbst, seines in eincr intelligibeln Ordnung der Dinge bestimmbaren Seins bewusst ist".

Das Ding an sich der empirischen Persönlichkeit liegt somit in ihrem Berufensein zum Sittlichen. In der Erscheinung äussert sich dies darin, dass das Sittengesetz dem Phänomenon, der nach aussen gewandten Seite der moralischen Persönlichkeit die Norm für Bestimmung und Beurteilung ihrer Ilandlungen vorschreibt. So bestimmt schon in der Erscheinung des Noumenon das Verhalten des Phänomenon. Die praktische Vernunft erlangt das Primat über die theoretische. Nicht die phänomenale Scito ist. Bestimmung des Menschen, sondern Ziel und Endzweck ist Erzichung zur Persönlichkeit. Erst mit dem aufgehenden Bewusst-

9) Romenkranz, VIII, 164.

x) a. a. 0. 165 . 
sein der Freiheit wird der Mensch seiner wahren Bestimmung gewiss. Auch diese Ideen von der moralischen Persönlichkeit als dem Ding an sich und dem Primat der praktischen Vornunft hat Fichto mit ganzer Energie zu den seinen gemacht und in seiner Weise behandelt und umgebildet. Das Faktum der intellektuellen Anschauung zu erklären „geschieht lediglich durch Aufweisung des Sittengesetzes in uns, in welchem das Ich als etwas über alle ursprüngliche Modifikation durch dasselbe Erhabenes vorgestellt, in welchem ihm ein absolutes, nur in ihm und schlechthin in nichts anderem begründetes Handeln angemutet und es sonach als ein absolut Thätiges charakterisiert wird. In dem Bewusstsein dieses Gesetzes ist die Anschauung der Selbstthätigkeit in der Freiheit begründet. „Ich habe das Leben in mir selbst und nehme es aus mir selbst" ${ }^{97}$ ). Das Ding an sich, das ursprüngliche, selbstthätige Ich erweist sich wirksam und als thatsächlich bestehend in dem Sittengesetz, welches Freiheit und Selbstthätigkeit realisiert, welches selbst clas einzig wahre Leben ist.

8. Mit dem Satz von der moralischen Persönlichkeit als dem Ding an sich, ist aber ein Satz durchbrochen, der in der Kritik der reinen Vernunft als unumstösslich galt und auch in der Transscendentalphilosophie gelten muss, nämlich der von der Unerkennbarkeit des „Ding-an-sich“. Dasselbe ist schlechterdings unerkennbar. Auch der intelligible Charakter erhält keinerlei näher bestimmende Erläuterung. Anders verhält es sich in der Kritik der praktischen Vernunft. Mit der Aufstellung des Sittengesetzes, der die Idee der Freiheit folgt, mit der Einführung der moralischen Persönlichkeit als autonomem Wesen, ist der Satz von der Unerkennbarkeit das Ding-an-sich umgestossen. Dies muss Kant zugeben, wenn auch unter mancherlei Drehungen und Wendungen. Es genügt, die bezeichnendsten Stellen aus der Kritik der praktischen Vernunft hierher zu setzen. „Ueber Erfahrungsgegenstïnde hinaus, also von Dingen als Noumenon wurde der spekulativen Vernunft alles Positive einer Erkenntnis mit völligem Rechte abgesprochen. Dagegen gibt das moralische Gesetz . . . ein schlechter-

97) Fichte, a. a. 0. 466. 
dings aus allen Datis der Sinnenwelt... unerklärliches Faktum an die Hand; das auf reine Verstandeswelt Anzeige gibt, ja diese sogar positiv bestimmt, und uns etwas von ihr, nämlich ein Gesetz erkennen lässt ${ }^{498}$ ), „das den Menschen nicht blos als zu einer reinen Verstandeswelt gehörig... gedacht (wie es nach der" Kritik der reinen Vernunft geschehen konnte), sondern ihn auch in Ansehung seiner Kausalität bestimmt, also unser Erkenntnis über die Grenzen des letzteren erweitert, welche Anmassung doch die Kritik der reinen Vernunft in aller Spekulation für nichtig erklärte $\left.{ }^{49}\right)$. Somit ist nicht nur ${ }_{n}$ die objektive Realität eines reinen Verstandesbegriffes ${ }^{\alpha}$ im Felde des Uebersinnlichen nachgewiesen, sondern auch sein Wesen "selbst in Absicht auf Noumenen hinreichend bestimmt".

9. Es erübrigt noch einen anderweitigen Punkt zu besprechen, der bei Kant nicht ganz klar zum Ausdruck kommt. Die völlige "Angemessenheit des Willens mit dem Sittengesetze", die Heiligkeit der Persönlichkeit, ist eine unabweissliche Forderung der Vernunft. Sie kann nur erreicht werden in einem unendlichen Progressus der allmählichen Vervollkommnung des Willens in der Realisierung des Sittengesetzes, da die kurze Zeit des endlichen Daseins nicht hinreicht, völlig Herr der Sinnlichkeit zu werden. Kant sucht diesen Progressus einerseits in einem jenseitigen Leben der einzelnen Persönlichkeit, andrerseits in einem stetigen Fortschritt der Jlenschheit. Beides nebeneinander dürfte überflüssig sein. Man könnte sagen: Wozu einen Fortschritt des Menschengeschlechts zur Verwirklichung des sittlichen, wenn diese in einer Fortdauer der Persōnlichkeit gewährleistet wird? Allerdings schliesst das eine das Andre nicht aus. Ist die Unsterblichkeit der Persōnlichkeit gewiss, so ist der allgemeine Fortschritt eine tröstliche Ermunterung für Gesetzgeber und Erzieher, alle Kraft einzusetzen, die Menschheit dieser Vollkommenheit immer näher zu führen. Aber in sonstiger Beziehung bleibt dieser Gedanke für die einzelne Persōnlichkeit belanglos, wie sie auch wegfallen könnte,

o" Posenkranz, VIII, 157.

T) Ebenda VIIl, 166.

Arctir L. Geacbictile \&. Pbllocoptie. X. 1. 
unboschadet des orstoren Gedankens. Anders aber steht es, wenn man dic Frage umkehrt: wozu eine Unsterblichkeit der Seele, wonn die Heiligkeit schon im Diesseits einstens erreicht wird? Wird dann ersterc nicht ïberflüssig?

Aber dieser Schluss und damit der Verlust des Gedankens der Unsterblichkeit wäre für die Persönlichkeit bedenklich. Zwei ernste, zum Nachdenken auffordernde Folgen bezeichnet Kant selbst, dic in Ermangelung dieses Postutals eintreten müssten, „entweder würde das moralische Gesetz von seiner Heiligkeit gänzlich abgewürdigt, indem man sich es als nachsichtig (indulgent) und so unsrer Behaglichkeit angemessen, verkünstelt ${ }^{* 100}$ ). Der Mensch würde ohne Aussicht, für seine Person jemals das Ziel erreichen zu können, den Mut verlieren, die strengen, unnachsichtlichen Forderungen des Sittengesetzes zu erfüllen, und die erhabene Strenge desselben zu mildern versuchen, und so es zu sich in die gemeine Wirklichkeit herabziehen. Der Gedanke des allgemeinen Fortschritts könnte keinen hinlänglichen Ersatz bieten, da er zu schwach ist, um die Spannkraft der sittlichen Energie auf gleicher Höhe zu erhalten. Das sittliche Streben des einzelnèn wäre angesichts der Erfahrung von der Art der Menschen fast ziellos, der Gedanke, dass Vollkommenheit des Einzelnen doch nie erreichbar sei, gerade zu lähmend und niederschmetternd. Andrerseits würde das Sittengesetz verlieren, da man "seinen Beruf und zugleich Erwartung zu einer unerreichbaren Bestimmung, nämlich einen erhofften, völligen Erwerb der Heiligkeit des Willens, spannt". Träumerische Naturen könnten leicht dazu verleitet werden, das Unmögliche für sich zu erhoffen und zu erstreben durch träumende Schwärmerei, die das sittliche Erkënnen verdunkelt und trübt, und so einen strengen, nur dem Vernunftgebot gehorchenden sittlichen Streben nur hiuderlich ist.

So ist dann einerseits die Lehre vom Fortschritt des Menschengeschlechts neben der von der Unsterblichkeit der Persönlichkeit von keiner grossen Bedeutung, da sie nicht imstande ist, das moralische Problem zu lösen, vielmehr dasselbe nur noch schwieriger

100) Ebenda VIII, 262. 
macht. Andrerseits tritt dieser Gedanke bei Kant thatsächlich nur vereinzelt auf. Er selbst glaubte mit Begeisterung an den Fortschritt des Menschengeschlechts. Doch ist dieser Gedanke kein Dogma bei ihm, nicht einmal ein Postulat, er tritt vielmehr nur gelegentlich auf. In dem Eingang des ersten Stückes der „Religion innerhalb der Grenzen der reinen Vernunft" nennt er sogar diesen Glauben neine heroische Meinung, die allein und vornehmlich in unsern Zeiten unter Philosophen und Pädagogen Platz gefunden hat" ... neine gutmüthige Voraussetzung der Moralisten von Seneka bis Rousseau"... gegen die „die Geschichte aller Zeiten gar zu mächtig spricht ${ }^{401}$ ).

Damit aber gewinnt das Postulat der Unsterblichkeit seine ganze, volle Bedeutung und Kraft wieder.

10. Der Begriff der Persönlichkeit ist, wio wir sehen, der Centralbegriff der Kant'schen Ethik, nach dem hin alles gravitiert. Es ist Kant besonders lieb und wichtig geworden. In den praktisch-ethischen Schriften hat er den kategorischen Imperativ verdrängt und dessen Stelle eingenommen. Aus ihm „dem Vernunftresen der Idee der Menschheit, der Idee der Menschheit als Zweck an sich", leitet er die verschiedensten Tugenden und Pflichten ab, wozu es für ihn gerade um dieser unbestimmten Allgemeinheit besonders geeignet schien. Es ist ein über allem Irdischen in unnahbarer Erhabenheit schwebendes Ideal. Schön, mächtig, herbe wie eine Juno Ludovisi. In der gänzlichen Loslösung dieses Ideals von allem Empirischen, in der scharfen Trennung ron Sinnlichkeit und Vernunft ist diese abstrakte Isolicrung begründet. Darin beruht gerade die Schönheit, die Macht und Stärke, aber auch die Schwäche des Ideals ${ }^{102}$ ).

101) Kant, Kebrbach S. 18.

${ }^{103} \mathrm{cf}$. auch das mir erst nachtrågliche Urteil Höffings in seiner Geschichte der veueren Pbilosopbie Leipzig 1896 über den Begriff der Persünlichkeit bei Kant: Fon dem Gekünstelten der Ableitung ahgeschen, bat Kant ein grosses und bedentungsrolles Prinzip ausgesprochen. Es ist das Prinzip der Persönlichkeit in seiner edelsten Form, ein Gedanke, welcher leben wird, wena Kants untollkommene und naturwidrige Begründung längst rergessen sein wird, ein Gedanke ron grossem, ethischen Werte, sowohl dem Autoritäsprinzip gegenüber, wenn dieses etwas mebr sein will, als ein erziebendes 
Wir sehen oben schon, wie schwer es Kant wurde eine Brücke zu finden zwischen Sinnlichkeit und Vernunft, um den Begriff der Persönlichkeit, das Sittengesetz auch für und auf die Erfahrung anwendbar zu machen. Es gelang ihm das nur mit Mähe und unvollkommen. Aber noch ein andrer . Nachteil fliesst aus dieser abstrakten Fassung des Sittengesetzes und des Begriffes der Persönlichkeit. Er soll als Regel und Maxime, als kategorischer Imperativ alles Handeln normieren. Aber in wie vielen Fällen wird er versagen und im Ungewissen lassen, gerade um seiner Unbestimmtheit und Abstraktheit willen? Es ist ein Schema, abstrakt und so fern allem Conkreten, so allgemein und unbestimmt, dass es manchmal nicht leicht sein wird für einen bestimmten Fall eine bestimmte Norm des Verhaltens herauszulesen.

Ist es ganz richtig ein Sittengesetz, ein Ideal der Persönlichkeit, das für individuelle Menschen Geltung haben soll; ganz ohne Rücksicht auf psychologische, anthropologische, nationale Thatsachen zu gestalten? Sind die Menschen so weit, dass ein ganz, allgemein gehaltenes, für alle "vernünftige Wesen überhaupt" zugeschnittenes Sittengesetż genügen könnte?

Prinzip, als auch gegenüber der Lehre von dem äusseren Nutzen und Glücke, die sich mit den Schalen begnügt und den Kern vergisst" (2. Band S. $93 \mathrm{f}$. ). 